\title{
Plume or bubble? mixed convection flow regimes and city-scale circulations
}

3
Hamidreza Omidvar ${ }^{1}$, Elie Bou-Zeid ${ }^{1, *}$, Qi Li ${ }^{2}$, Juan-Pedro Mellado $^{3}$, Petra

Klein $^{4}$

${ }^{1}$ Department of Civil and Environmental Engineering, Princeton University, USA

${ }^{2}$ School of Civil and Environmental Engineering, Cornell University, USA

${ }^{3}$ Department of Physics, Division of Aerospace Engineering, Universitat Politécnica de Catalunya, Barcelona, Spain ${ }^{4}$ School of Meteorology, University of Oklahoma, USA

*Correspondence: Elie Bou-Zeid, ebouzeid@princeton.edu

\section{Abstract}

Large-scale circulations around a city are co-modulated by the urban heat island and by regional wind patterns. Depending on these variables, the circulations fall into different regimes ranging from advection dominated (plume regime) to convection driven (bubble regime). Using dimensional analysis and large eddy simulations, this study investigates how these different circulations scale with urban and rural heat fluxes, as well as upstream wind speed. Two dimensionless parameters are shown to control the dynamics of the flow: (1) the ratio of rural to urban convective velocities that contrasts urban and rural buoyancy fluxes, and (2) the ratio of bulk inflow velocity to the thermal convection velocity in the rural area. Finally, the vertical flow velocities over the rural and urban areas are used to develop a criterion for categorizing different large-scale circulations into plume, bubble or transitional regimes. The findings have implications for city ventilation since bubble regimes are expected to trap pollutants, as well as for the scaling of canonical mixed-convection flows. 


\section{Introduction}

Mixed convection occurs when both natural and forced convection processes act together

27 to transfer heat, for example from a hot surface patch to the surrounding fluid in the presence of a

28 wall-parallel flow. The applications of mixed convection range from small-scale problems such as

29 cooling of industrial electronic chips (Akbarinia \& Behzadmehr 2007) to large scale flows such as

30 ventilation of buildings or cities (De Foy et al. 2006; Venko et al. 2014). In general, understanding

31 mixed convection flow processes is more challenging than natural or forced convection due to the

32 simultaneous and interacting effects of buoyancy and advection on flow dynamics. Probably the

33 most well-known similarity theory dealing with mixed convection for wall-bounded flows is the

34 Monin-Obukhov similarity theory that provides a "buoyancy correction" to the classic logarithmic

35 laws of momentum and heat transfer over a flat homogeneous wall (Monin \& Obukhov 1954).

36 Nevertheless, mixed convection in turbulent flows remains a scarcely understood process that is

37 almost absent from most standard heat transfer textbooks (Bejan 1993; Bergman et al. 2011). The

38 reference on the subject of turbulent mixed convection dates from 1986 (English translation 2 years

39 later Petukhov et al. (1988)), before the emergence of modern flow simulation techniques.

Large-scale circulation around cities is an important example, among many, of a mixed

41 convection problem in the environment. Due to urbanization of the land surface and excess

42 anthropogenic heat emission, urban areas are generally hotter than their surrounding rural areas, a

43 phenomenon called the Urban Heat Island (UHI) (Oke 1982). Therefore, parcels of air heated over

44 the city become lighter than their surroundings and lift up. As they rise to the top of the

45 Atmospheric Boundary Layer (ABL), they may be advected downstream by the background wind

46 over the city. However, if the streamwise mean wind speed is weak, the parcels will be trapped in

47 a thermal recirculation bubble and advected back to the city. The mechanism responsible for that 
48 thermal recirculation is the horizontal surface convergence into the city from the surroundings to

49 replace the rising hot urban air, creating low pressure around the city. When this rising urban air

50 encounters the inversion at the top of the ABL, it diverges outwards and is then "sucked down" by

51 this urban-fringe low-pressure zone to complete a thermal circulation cell. This is very similar to

52 a Rayleigh-Bénard cell that is locked in place by a horizontal temperature contrast at the surface.

53 However, under high wind conditions, streamwise advection destroys these convergence and

54 pressure spatial patterns and transports parcels downstream of the city. While in the former weak

55 wind case a bubble (or dome) shaped circulation is formed around the city, in the latter strong wind

56 case a plume of urban air forms and extends downwind (see sketch in Figure 1). Under bubble

57 circulation patterns, the pollutants and heat lofted from the city are constantly being recirculated

58 into the city, deteriorating urban ventilation and environmental quality. This case is usually

59 associated with poor air quality conditions (Klein 2012). On the other hand, plume formation could

60 indicate more effective removal of urban emissions and the replenishment of the city with fresh

61 air by advection, improving environmental quality inside the city. However, in this case, the plume

62 of air transfers heat, moisture, aerosols and other pollutants to downstream regions, deteriorating

63 environmental quality in rural areas in the lee of cities, as well as potentially increasing the chance

64 of precipitation in these downstream areas (Changnon 1979; Shepherd 2005).

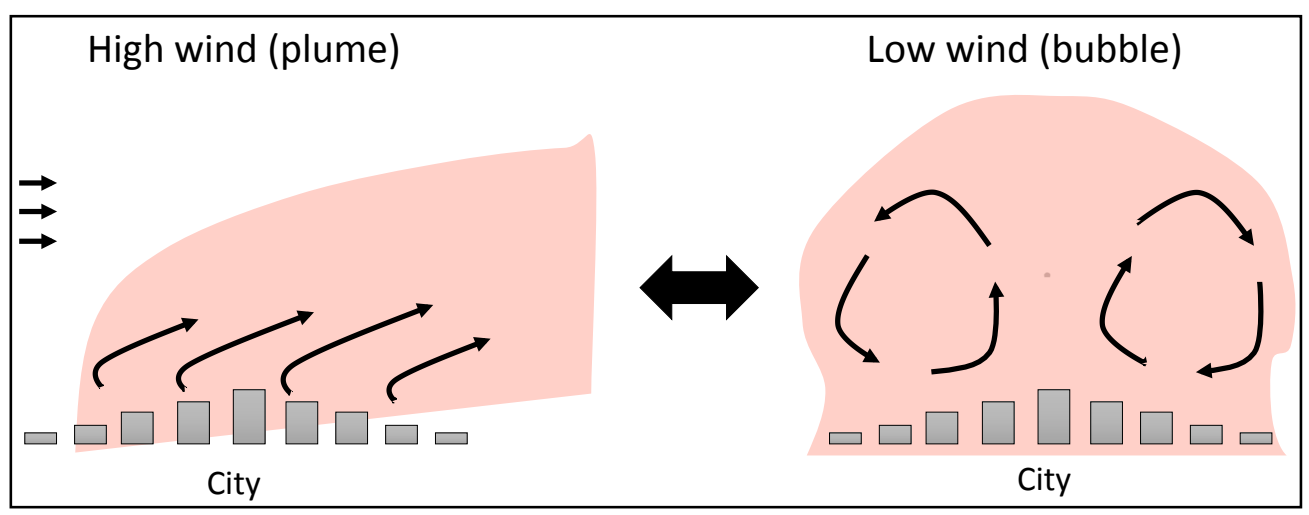

Figure 1. Bubble and plume regimes of city-scale circulations 
Previous research studies on this topic have mostly focused on the natural convection,

68 Rayleigh-Bénard-like regime, and range from laboratory experimental investigations to analytical

69 and numerical modeling studies (Ahlers et al. 2009; Fan et al. 2016, 2017; Kurbatskii \&

70 Kurbatskaya 2016; Ryu et al. 2013). The forced convection limit was also considered, for example

71 with passive scalars emitted from the urban core. These studies investigate the flow dynamics over

72 cities under high wind conditions, mostly focusing on how the roughness elements (buildings)

73 influence flow and transport dynamics at smaller scales, i.e. building to neighborhood scales

74 (Hataya et al. 2014; Li \& Bou-Zeid 2019; Llaguno-Munitxa \& Bou-Zeid 2018; Mochida et al. 75 2008).

transitions from a natural convection regime to a mixed convection regime and then to a forced convection regime as the wind speed gradually increases (relative to the velocity scale associated with surface heat flux to be defined later in this paper). This hinders our understanding and ability to model the full range of atmospheric conditions encountered in the real world. In particular, the

81 following research questions remain open and will be the focus of this paper:

82 i) How do different atmospheric circulation regimes scale with urban and rural surface heat 83 fluxes and wind velocity over the city?

84 ii) What are the critical values of the scaling parameters identified in (i) that characterize the flow 85 transitions from a bubble to a plume regime?

86 In this paper, we bridge this gap using large eddy simulation (LES). First, using 87 dimensional analysis, we derive two non-dimensional parameters that are expected to control the 88 dynamics of city-scale circulations and explain the behavior of ABL under various wind and UHI 89 strengths (section 2). Then, we verify the validity of our dimensional analysis results using LES 
90 (sections 3 and 4). In section 5, we use these two dimensionless parameters to categorize different

91 ABL circulations over cities as bubble, plume and transitional regimes. Finally, we discuss the

92 findings implications in section 6.

\section{Dimensional analysis}

94 A primary aim of this study is to develop a general theoretical framework for assessing the 95 relative roles of natural and forced convection in this class of problems, and how their relation

96 modulates flow features. We start with an overview of the dimensional thermal and geometric

97 parameters that are relevant in this problem (superscripts $u$ and $r$ refer to urban and rural areas

98 respectively): (1) horizontal extent of the city, $L_{c}(\mathrm{~m})$; (2) ABL height, $z_{i}(\mathrm{~m})$; (3) and (4)

99 momentum roughness length of the urban and rural areas, $z_{0, u}(\mathrm{~m})$ and $z_{0, r}(\mathrm{~m})$ respectively; (5) the

100 spatially-averaged (bulk) mean inflow speed, $M\left(\mathrm{~m} \mathrm{~s}^{-1}\right)$; and (6) and (7) buoyancy fluxes from the

101 urban and rural surfaces, $\frac{g}{\theta_{0}}\left(\overline{\theta^{\prime} w^{\prime}}\right)_{u}$ and $\frac{g}{\theta_{0}}\left(\overline{\theta^{\prime} w^{\prime}}\right)_{r}\left(\mathrm{~m}^{2} \mathrm{~s}^{-3}\right)$ respectively, where $g=9.81\left(\mathrm{~m} \mathrm{~s}^{-2}\right)$

102 is the gravitational acceleration; $\theta_{0}(\mathrm{~K})$ is a reference temperature (taken as $300 \mathrm{~K}$ in this paper);

103 and $\theta^{\prime}$ and $w^{\prime}$ are the temperature and vertical velocity turbulent perturbations, respectively (their

104 covariance is the kinematic vertical heat flux). As output, we are interested in a given velocity

105 component $(u, v$, or $w)$ that we will denote as $(8) u_{o}\left(\mathrm{~m} \mathrm{~s}^{-1}\right)$, though one could also be interested in

106 the temperature field or other flow variables. Note that surface temperatures and thermal roughness

107 lengths are not invoked since we use the buoyancy fluxes directly. 
Using the definition of the convective velocity scale $w_{*}=\left(\frac{g}{\theta_{0}} \overline{\theta^{\prime} w^{\prime}} z_{i}\right)^{1 / 3}$ (Deardorff 1970),

109 a dimensionless formulation of the problem with six non-dimensional parameters ( 8 variables -2

110 dimensions) can be constructed

$$
\frac{u_{o}}{w_{*, r}}=f\left(\frac{z_{i}}{L_{c}}, \frac{z_{0, u}}{z_{0, r}}, \frac{z_{0, u}}{L_{c}}, \frac{M}{w_{*, r}}, \frac{w_{*, u}}{w_{*, r}}\right) .
$$

113 properties of the city and rough walls; in this paper we keep them constant (at typical values) in 114 order to focus on the dynamical effects of advection and convection encoded in the last two 115 parameters. However, we are not suggesting that these geometric parameters, kept fixed here, are 116 not important. $z_{i} / L_{c}$ is the ratio of the two bulk (outer) length scales of this problem; it encodes 117 the aspect ratio (height to the horizontal scale) of the secondary circulations, and it was shown in 118 Niino et al. (2006) to determine the type of the bubble circulation patterns. $z_{0, u} / z_{0, r}$ is the ratio of 119 the surface roughness length (inner) scales of the problem representing the change in surface stress 120 (see, for example, Kimura (1976), Sawai 1978 and Bou-Zeid et al. (2004) for an illustration of 121 roughness transition effects on the flow). $z_{0, u} / L_{c}$ encodes the relation between the inner and outer 122 scales and might not be relevant if the scale separation in the turbulent spectrum is large (very high 123 Reynolds number). Other potentially important parameters could be also formulated to account, 124 for example, for the shape of the urban region since here we only consider square cities (circular 125 or ellipsoidal cities are also common and previous studies indicate that this shape can have an 126 impact on bulk circulation patters, e.g. Fan, Li, \& Yin, 2018). While future studies should 127 investigate the impact of the geometric setup of the problem (Sawai 1978), here we elect to focus 128 on the mixed convection dynamics. 
With this focus, equation (1) is therefore simplified to

$$
\frac{u_{o}}{w_{*, r}}=f\left(\frac{M}{w_{*, r}}, \frac{w_{*, u}}{w_{*, r}}\right)
$$

131 Note here that we impose a similar $z_{i}$ for both rural and urban areas; therefore, the ratio of

132 convective velocities can be further simplified to $\left(\left(\overline{\theta^{\prime} w^{\prime}}\right)_{u} /\left(\overline{\theta^{\prime} w^{\prime}}\right)_{r}\right)^{1 / 3}$. However, for consistency

133 with other dimensionless parameters in equation (2), we will keep expressing the ratio as one of

134 velocity scales. In addition, we only consider positive values of $w_{*, r}$ and $w_{*, u}$ corresponding to

135 daytime convective conditions for both rural and urban areas with positive heat fluxes (where the

136 heat flux over the urban area is higher than over the rural area due to the UHI). Potentially

137 interesting conditions, which we do not consider here, could occur when $w_{*, r}<0$ but $w_{*, u}>0$ or

138 when both are negative.

139 Depending on the relative magnitude of the two input dimensionless parameters in equation

140 (2), we hypothesize (and confirm in section 4) that three scenarios will emerge

141 a) When $\frac{M}{w_{*, r}} \gg \frac{w_{*, u}}{w_{*, r}}$, equation (2) can be reduced to $\frac{u_{o}}{w_{*, r}}=f\left(\frac{M}{w_{*, r}}\right)$. In this scenario, the

142 dominant factor is forced advection from the inflow, and convection due to surface heat fluxes can

143 be neglected.

144 b) When $\frac{M}{w_{*, r}} \ll \frac{w_{*, u}}{w_{*, r}}$, equation (2) can be reduced to $\frac{u_{o}}{w_{*, r}}=f\left(\frac{w_{*, u}}{w_{*, r}}\right)$. In this scenario, the ABL

145 is close to the free (natural) convection limit, the circulations are mostly thermally driven, and 146 advection due to $M$ plays no role. 
147 c) When $\frac{M}{w_{*, r}} \sim \frac{w_{*, u}}{w_{*, r}}$, the ABL experiences a mixed convection, and both input parameters on

148 the right hand side of equation (2) should be considered.

149 We should also here point out that the velocity ratios $M / w_{*, r}$ and $M / w_{*, u}$ can each be related to

150 a distinct Richardson numbers $R i$ (each of these ratios $\sim R i^{-1 / 3}$ ). However, we find that our

151 dimensionless formulation is more informative about the physics of the problem (for example in

152 formulating these three scenarios) and we will thus not use the conventional measure of stability

153 related to $R i$.

\section{$154 \quad 3 \quad$ Large eddy simulations}

155 In the current LES model, the city blocks (as groups of buildings) are resolved using the 156 Immersed Boundary Method (IBM) (Peskin, (2002); the exact implementation and validation can

157 be found in Li, Bou-Zeid, \& Anderson, (2016) and Li et al. (2016). To obtain the velocity and 158 temperature fields, the spatially-filtered incompressible continuity and Navier-Stokes equations 159 using the Boussinesq approximation, in conjunction with the advection-diffusion equation for 160 temperature, are solved as follows:

$$
\frac{\partial \tilde{u}_{i}}{\partial t}+\tilde{u}_{j}\left(\frac{\partial \tilde{u}_{i}}{\partial x_{j}}-\frac{\partial \tilde{u}_{j}}{\partial x_{i}}\right)=-\frac{1}{\rho} \frac{\partial \tilde{p}^{*}}{\partial x_{i}}-g \frac{\tilde{\theta}^{\prime}}{\theta_{0}} \delta_{i 3}-\frac{\partial \tau_{i j}}{\partial x_{j}}+\tilde{F}_{i}+\tilde{B}_{i}
$$

$$
\frac{\partial \tilde{\theta}}{\partial t}+\tilde{u}_{j} \frac{\partial \tilde{\theta}}{\partial x_{j}}=-\frac{\partial \pi_{j}}{\partial x_{j}}
$$


164 where the tilde $(\sim)$ represents filtered quantities (from now on, we omit the tilde for simplicity since

165 all variables are filtered); $u_{i}$ is the velocity vector in a Cartesian coordinate system (where $i$ and $j$

$166=1,2$, or 3 ); $t$ is time; $\rho$ is the air density; $F_{i}$ is the immersed boundary force imposed by the

167 buildings; $B_{i}$ is a body force (e.g. the mean pressure gradient force driving the flow, which is

168 needed for periodic boundary conditions but not when a domain inflow is imposed); $\theta$ is the

169 potential temperature; $\pi_{j}$ is the sub-grid scale (SGS) heat flux; $\tau_{i j}$ is the anisotropic part of SGS

170 stress tensor; and $p^{*}$ is the modified pressure computed as:

$$
\tilde{p}^{*}=\tilde{p}+(1 / 3) \rho \sigma_{k k}+(1 / 2) \rho \tilde{u} \tilde{u}_{j},
$$

172 where $p$ is the total pressure, and $\sigma_{k k}$ is the trace of the full SGS stress tensor. Note that the 173 Coriolis force is not included.

174 The LES model uses a scale-dependent Lagrangian dynamic model to calculate the SGS 175 stress (Bou-Zeid et al. 2005), and a constant SGS Prandtl number of 0.4 to infer the SGS diffusivity 176 and compute the SGS heat flux (Li 2016).To compute the vertical derivatives on a staggered 177 uniform grid, a second-order finite difference method is used. A pseudo-spectral differentiation 178 scheme is adopted for horizontal derivatives (see Li, Bou-Zeid, \& Anderson, (2016) for details on 179 implementation with the IBM method to avoid the Gibbs phenomenon). Finally, an explicit 180 second-order Adam-Bashforth scheme is used for the time advancement. More information and 181 validation of the basic code can be found in other references (Bou-Zeid et al. 2005; Huang \& Bou182 Zeid 2013; Shah \& Bou-Zeid 2014). 


\section{3}

184 194 limitations, resulting in a reduced accuracy in representing the small-scale eddies in between 195 buildings. However, our analyses do not examine this small-scale turbulence but rather focus only 196 on the large, city-scale eddies and flow patterns that will not be significantly affected by the 197 resolution of each block. A grid sensitivity to demonstrate this assertion is shown in Appendix A.

\subsection{Domain configuration and high-resolution simulation setup}

Figure 2 shows the domain setup, where the city consists of 36 cubes each representing a full city block. The domain size is $5 \mathrm{~km} \times 4.5 \mathrm{~km} \times 0.417 \mathrm{~km}$ in the $x$ (streamwise), $y$ (crossstream) and $z$ (vertical) directions, respectively, and the corresponding baseline number of grid points is $288 \times 256 \times 48$. This leads to a grid cell size of $\Delta x=\Delta y=2 \Delta z=17.4 \mathrm{~m}$. The city is a square with a side of $885 \mathrm{~m}$, with one side normal to the incoming inflow velocity. Each cube is resolved using 6 grid points in each direction (minimum needed for an adequate representation of the flow in this code as demonstrated in Tseng et al. 2006), which result in a city block size of $104 \mathrm{~m} \times 104 \mathrm{~m} \times 52 \mathrm{~m}$. The width of the street between two adjacent cubes is $52 \mathrm{~m}$ ( 3 grid points), while the ratio of the domain height to the building height is around 8 (which is sufficient based on Li et al. 2016). The resolution of each street or building is kept low due to computational power 


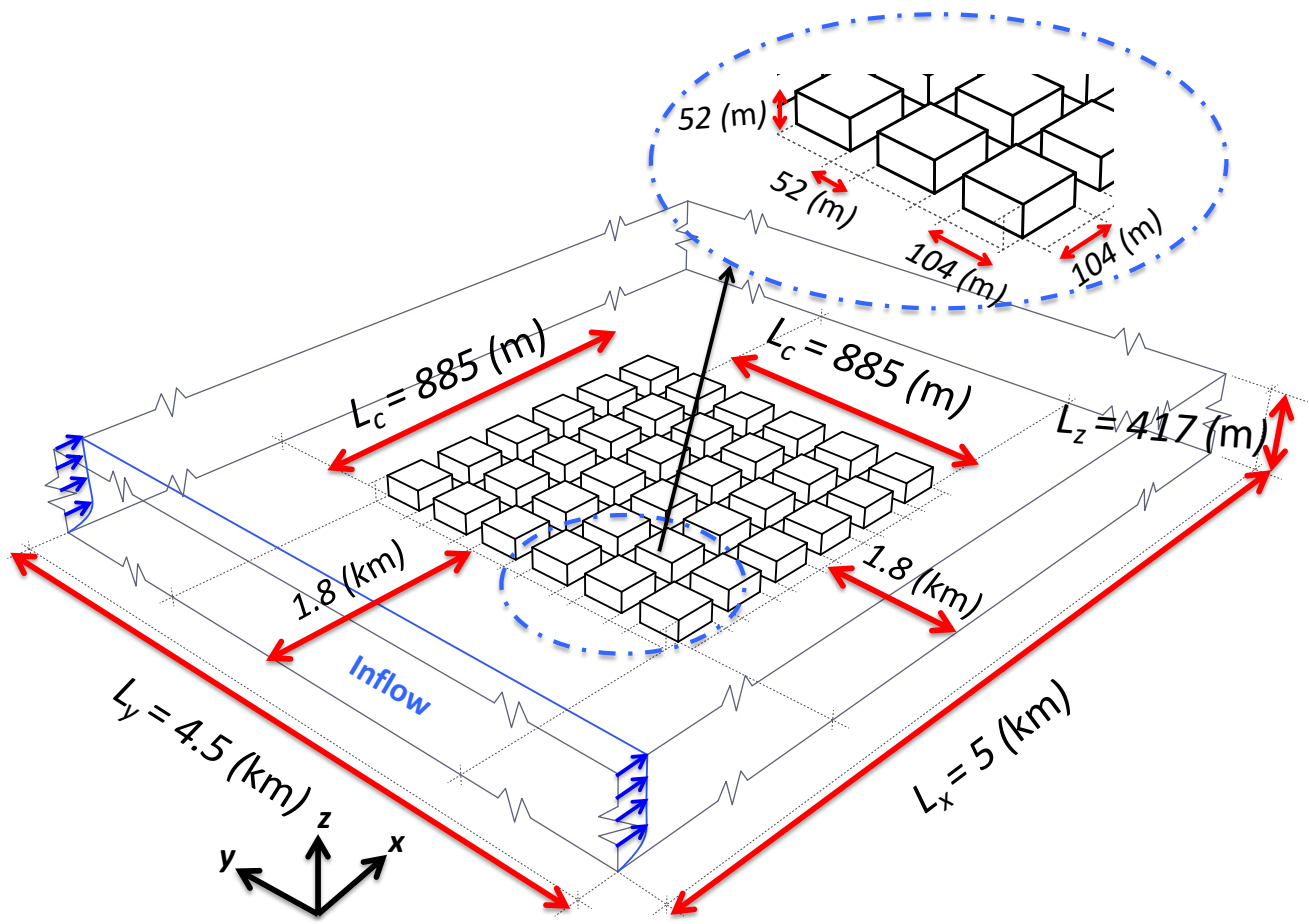

Figure 2. Simulation setup and geometric dimensions. In addition, $z_{0, r}=0.1 \mathrm{~m}$ is used for rural terrain, while a roughness of $0.01 \mathrm{~m}$ is considered for the individual facets of the blocks. The effective $z_{0, u}$ of the urban terrain will be higher since it would also include the building drag resolved by the immersed

A zero-shear stress, no penetration boundary condition at top of the domain is imposed for 204 the velocities. Additionally, a temperature inversion layer with strength of $0.08 \mathrm{~K} \mathrm{~m}^{-1}$ is imposed 205 in the top $20 \%$ of the domain. In order to ensure this inversion layer is maintained (and is not 206 eroded by the rising thermals), at each time step, the average temperature increase of the domain 207 below the inversion is calculated and added to the temperature above the inversion layer. That is, 208 the inversion layer is warmed up artificially at the same rate as the other parts of the domain, 209 leading to a constant boundary layer height. Furthermore, in order to prevent wave reflection at 210 the top of the domain in this stably stratified inversion layer, the velocities are damped in the top 
$21185 \%$ of the domain using the Raleigh damping method (Klemp \& Lilly 1978). This vertical setup

212 implies that the actual boundary layer depth is $z_{i}=0.8 L_{z}=333 \mathrm{~m}$.

213 The boundary conditions in the $y$ direction are periodic for velocities and temperature in

214 all simulations. Therefore, the domain size in the $y$ direction is made large enough to prevent the

215 circulations at the lateral edges of the city from interacting with each other through the periodic

216 boundaries (details of domain sensitivity analysis are shown in Appendix C). An inflow boundary

217 condition for the velocity is imposed in the $x$ direction (except for the simulations where $M=0$

218 where periodic boundary conditions in $x$ are imposed). The boundary condition in the $x$ direction

219 for temperature is an inflow for the simulations when $M / w_{*, r}$ is larger or on the order of

$220 w_{*, u} / w_{*, r} ;$ however, it is periodic for the simulations where $M / w_{*, r} \ll w_{*, u} / w_{*, r}$ (in this limit,

221 the convection processes are dominant and the Monin-Obukhov Similarity Theory (MOST) is no

222 longer valid for the re-scaling of the temperature inflow that will be discussed in this section). At

223 the end of the domain in the $x$ direction, when an inflow is used (we will discuss how the inflow

224 is generated later), we impose a buffer region consisting of $y-z$ planes that, at each time step,

225 interpolates/recycles the outflow solutions of velocities and temperature to the imposed/desired

226 inflow values (Lund et al. 1998; Spalart 1988). The length of this buffer area is approximately

$2271 / 32$ of the domain length in the $x$ direction. To make sure that the wake of the flow downstream

228 of the city (especially for high wind simulation cases) does not perturb the interpolation in the

229 buffer region, the city is located slightly upstream of the center of the domain in the $x$ direction, at

$2301.8 \mathrm{~km}$ from the inflow boundary and at $2.315 \mathrm{~km}$ from the outflow boundary. The distance of the

231 city edge to the $y$-boundaries is also $1.8 \mathrm{~km}$. The most appropriate length scale for normalizing

232 these geometric scales is the depth of the boundary layer $(333 \mathrm{~m})$. 
The inflows for velocities and temperature are generated in precursor simulations with

234 periodic boundary conditions in both $x$ and $y$ directions. The surface heat flux and momentum

235 roughness length imposed in the precursor simulations are set equal to their values over rural areas

236 in the main simulations to represent an infinitely homogeneous upstream fetch. In order to

237 minimize the number of precursor runs for inflow generation, for each unique value of rural heat

238 flux, only one main inflow is generated; subsequently the inflow velocities and temperatures are

239 re-scaled to modify the inflow bulk velocity and produce simulations with a different $M / w_{*, r}$.

240 The details on rescaling the inflow velocity and temperature are discussed in Appendix B, but we

241 note that this rescaling will have a minimal impact on our results because (i) the inflow is allowed

242 to evolve over a distance $\approx 5$ times the boundary layer depth $(1800 \mathrm{~m} / 333 \mathrm{~m})$ inside the main

243 domain to further adjust to the upstream rural surface before it meets the city (Bou-Zeid et al.

244 2004), and (ii) regardless of the rescaling results, the $M$ used in the analyses is the one actually

245 attained and computed just upstream of the city in the main domain.

246 Recall that for all the simulations we set that the momentum roughness length for rural area

247 to $0.1 \mathrm{~m}$ and that of the facets of the blocks (i.e. walls, roofs, and streets) to $0.01 \mathrm{~m}$. In addition,

$248 z_{0, h}$ is taken as $1 / 10$ of the momentum roughness length to approximate rough rural surfaces for

249 inflow rescaling (Eq. 12 in Appendix B), but the value of $z_{0, h}$ is not needed in the wall model of

250 the LES since we prescribe the heat flux. We impose the surface heat flux for rural

$251 \quad\left(H_{r}=\rho c_{p}\left(\overline{\theta^{\prime} w^{\prime}}\right)_{r}\right)$ and urban $\left(H_{u}=\rho c_{p}\left(\overline{\theta^{\prime} w^{\prime}}\right)_{u}\right)$ areas, with the urban area heat flux taken to

252 be greater than the rural area heat flux to represent UHI conditions ( $\rho$ and $c_{p}$ are the density and 253 heat capacity of the air). In addition, in the urban areas, heat fluxes are imposed on the horizontal 
254 surfaces (ground surface and roof, but not walls) of the buildings in order to represent conditions 255 around solar noon.

\section{$256 \quad 3.2$ Simulation scenarios and methodology}

To answer the two questions on city-scale circulations overviewed in the introduction, we

258 adopt the following methodology:

259 i) To test the two scaling parameters proposed in the dimensional analysis section and the general

260 validity of that analysis, we conduct high-resolution large eddy simulations based on the setup

261 introduced in the previous section. These analyses help us understand how important each of the

262 scaling parameters is under different circulation regimes (a to $\mathrm{c}$ in section 2), and how the dynamics

263 of these circulations transition between the different regimes. Details and results of these

264 simulations are discussed next and in section 4.

265 ii) To propose a generalizable categorization of the circulations into bubble, transitional and 266 plume regimes, we need a larger suite of simulations to cover the entire parameter space.

267 Therefore, in section 5, we introduce a suite of a lower-resolution simulations that fully and finely

268 span that parameter space for $M / w_{*, r}$ and $w_{*, u} / w_{*, r}$. These simulations then enable us to classify

269 the flows into the three regimes and to identify a parameter that can a priori predict the resulting

270 flow regime.

271 First we focus on testing the scaling hypothesized in equation (2). To that end, we 272 conducted eight simulations detailed in Table 1:

273 a) When $M / w_{*, r} \sim w_{*, u} / w_{*, r}$, four simulation cases are conducted (cases 1 to 4 in Table 1). Case

2741 is the base case in this regime with $M / w_{*, r}=0.96$ and $w_{*, u} / w_{*, r}=1.4$. Then cases 2,3 , and 4

275 are constructed by changing the values of $M, w_{*, r}$, and $w_{*, u}$ in order to obtain: 
(i) Case 2: maintain both ratios $M / w_{*, r}$, and $w_{*, u} / w_{*, r}$ equal to the base case but with different dimensional inputs: the results should be identical to the base case if our hypothesis is correct, and the flow is controlled only by these two dimensionless parameters.

(ii) Case 3: only $w_{*, u} / w_{*, r}$ is changed from the base case to illustrate that the results are different from the base case and that this ratio is consequential.

(iii) Case 4: only $M / w_{*, r}$ is changed from the base case to show that the results are different from the base case and that this ratio is also consequential.

283 These cases allow us to show that when both dimensionless parameters are on the same order, they 284 both impact the flow dynamics and are important to scale the problem.

b) When $M / w_{*, r} \gg w_{*, u} / w_{*, r}$, we conducted 2 simulations (case 5 and 6 in Table 1). We consider case 5 as the base case of this limit with $M / w_{*, r}=15.9$ and $w_{*, u} / w_{*, r}=1.4$. Case 6 is constructed 287 by keeping $M / w_{*, r}$ the same as the base case but changing $w_{*, u} / w_{*, r}$. The two simulation are 288 shown to be similar to demonstrate that in this limit, $M / w_{*, r}$ is the only controlling non289 dimensional ratio and changes in $w_{*, u} / w_{*, r}$ are inconsequential.

290 c) Finally, the limit where $M / w_{*, r} \ll w_{*, u} / w_{*, r}$ is examined in cases 7 and 8 in Table 1. Case 7 is 291 the base case with $M / w_{*, r}=0$ and $w_{*, u} / w_{*, r}=1.4$, while case 8 has the same $w_{*, u} / w_{*, r}$ as the 292 base case, but $M / w_{*, r}=0.1$. The results are shown to be practically identical, demonstrating that $293 M / w_{*, r}$ is irrelevant and the ratio of the convective velocity scales dominates the dynamics in this 294 limit of natural convection. 
Table 1. Simulation cases: numbers underlined and in bold are the ones that were modified from the base case for each regime.

\begin{tabular}{|c|c|c|c|c|c|c|}
\hline $\begin{array}{c}\text { Case } \\
\text { Number }\end{array}$ & Description & $M\left(\mathrm{~m} \mathrm{~s}^{-1}\right)$ & $\begin{array}{l}H_{u}\left(\mathrm{~W} \mathrm{~m}^{-2}\right) \\
\left(w_{*, u}\left(\mathrm{~m} \mathrm{~s}^{-1}\right)\right)\end{array}$ & $\begin{array}{l}H_{r}\left(\mathrm{~W} \mathrm{~m}^{-2}\right) \\
\left(w_{*, r}\left(\mathrm{~m} \mathrm{~s}^{-1}\right)\right)\end{array}$ & $\frac{M}{w_{*, r}}$ & $\frac{w_{*, u}}{w_{*, r}}$ \\
\hline $\begin{array}{c}1 \\
\text { (Base) }\end{array}$ & \multirow{4}{*}{$\frac{M}{w_{*, r}} \sim \frac{w_{*, u}}{w_{*, r}}$} & 1 & $\begin{array}{c}300 \\
(1.50) \\
\end{array}$ & $\begin{array}{c}100 \\
(1.04) \\
\end{array}$ & 0.96 & 1.4 \\
\hline 2 & & 0.79 & $\begin{array}{c}150 \\
(1.19) \\
\end{array}$ & $\begin{array}{c}50 \\
(0.83) \\
\end{array}$ & 0.96 & 1.4 \\
\hline 3 & & 0.79 & $\begin{array}{c}300 \\
(1.50)\end{array}$ & $\begin{array}{c}50 \\
(0.83)\end{array}$ & 0.96 & $\underline{1.8}$ \\
\hline 4 & & 1 & $\begin{array}{c}150 \\
(1.19)\end{array}$ & $\begin{array}{c}50 \\
(0.83)\end{array}$ & $\underline{1.2}$ & 1.4 \\
\hline $\begin{array}{c}5 \\
\text { (Base) }\end{array}$ & \multirow{2}{*}{$\frac{M}{w_{*, r}} \gg \frac{w_{*, u}}{w_{*, r}}$} & 16.5 & $\begin{array}{c}300 \\
(1.50)\end{array}$ & $\begin{array}{c}100 \\
(1.04)\end{array}$ & 15.9 & 1.4 \\
\hline 6 & & 16.5 & $\begin{array}{c}100 \\
(1.04)\end{array}$ & $\begin{array}{c}100 \\
(1.04)\end{array}$ & 15.9 & $\underline{1}$ \\
\hline $\begin{array}{c}7 \\
\text { (Base) }\end{array}$ & \multirow{2}{*}{$\frac{M}{w_{*, r}} \ll \frac{w_{*, u}}{w_{*, r}}$} & 0 & $\begin{array}{c}300 \\
(1.50)\end{array}$ & $\begin{array}{c}100 \\
(1.04)\end{array}$ & 0 & 1.4 \\
\hline 8 & & 0.1 & $\begin{array}{c}150 \\
(1.19) \\
\end{array}$ & $\begin{array}{c}50 \\
(0.83) \\
\end{array}$ & $\underline{0.12}$ & 1.4 \\
\hline
\end{tabular}

\section{Results}

\subsection{City-scale circulations: flow characteristics}

Figure 3 depicts a pseudocolor plot of the time-averaged streamwise velocity $\langle u\rangle_{t}$ (brackets

303 denote time averaging; more details will be provided in section 4.2) in a $x-z$ slice that crosses the 304 mid-point of the city (at $y / L_{c}=2.5$ ). Three plots illustrate unambiguously the existence of the three 305 distinct circulation regimes: (a) plume regime (case 5), (b) transitional regime in between plume 
306 and bubble (case 1), and (c) bubble regime (case 7). For the plume case (from Figure 3a), the flow

307 over the city is deflected upward as it reaches the city, and then it subsides in the downstream area.

308 The plot suggests that the city influences the flow up to 3 times the city height $(H)$. Then, above

309 this height, the city effects on the flow dynamics become minimal. The flow velocity is generally

310 slower downstream of the city, with a small recirculation zone behind the city. In the bubble case,

311 Figure 3c, two symmetric main circulations can be seen on either side of the city with comparable

312 strengths. The horizontal distance over which each of these circulations extends is approximately

313 equal to the city size. In addition, there are smaller and weaker secondary circulations further away

314 from the city (both up and downstream of the city) with smaller horizontal scales. The flow pattern

315 in the transitional case, Figure $3 b$, is a fusion of the flow characteristics of the plume and bubble

316 regimes. In this case, there is a general horizontal direction for the flow (from left to right in Figure

$3173 \mathrm{~b}$ ); however, above the city, the velocity is mainly upward indicating that thermal convection

318 overcomes advection and lifts the warm air parcels to produce a unique circulation around the city. 

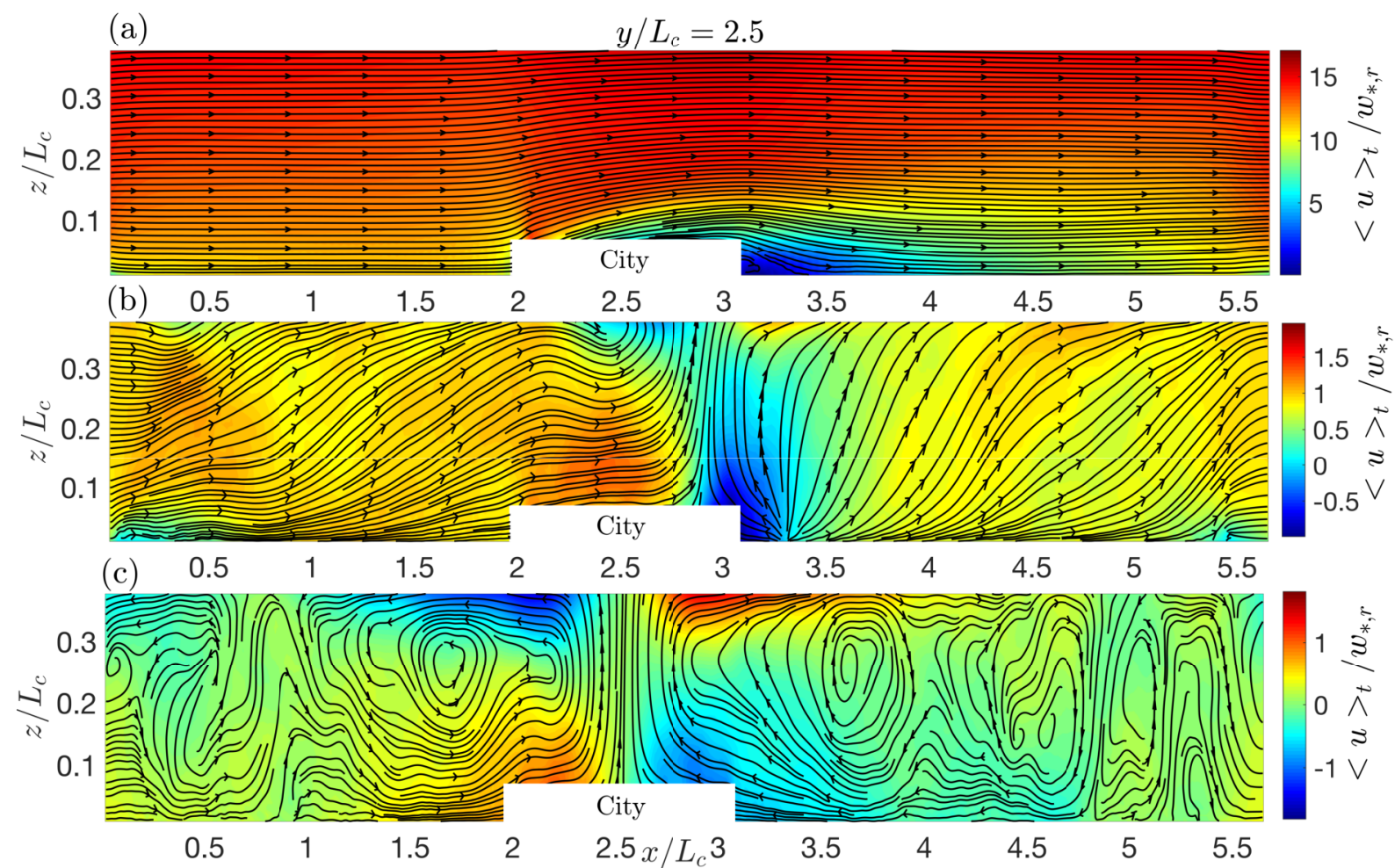

Figure 3. Pseudocolor plots of time-averaged normalized streamwise velocity $\langle u\rangle$ over $x$ - $z$ slices for the

321 cases of plume (case 5) (a), transitional (case 1) (b), and (c) bubble regimes (case 7). The inversion layer

322 (which starts at $0.8 L_{z}$ ) is excluded from the plots. The white masked area contains both city blocks (solid space) and streets (fluid space).

Figure 4 shows the pseudocolor maps of the vertical velocity in $x-y$ slices for the bubble

325 regime case (also averaged in time). These slices are shown for three different heights: $z / H=1.5$,

$326 z / H=4$, and $z / H=5.8$. This figure reveals the 3D structure of the circulations around the city. For

327 all heights, a high vertical velocity region above the urban area can be seen. At low elevations

$328(z / H=1.5)$, there is a convergence zone above the city, while for higher elevations $(z / H=5.8)$ a

329 divergence zone can be seen over the urban region. For intermediate heights $(z / H=4)$, the flow is

330 less structured; however, the main circulation around the city is still very clear. For $z / H=1.5$, the

331 maps of vertical velocity match the city topography, and strong upwelling thermals (red bands in

332 Figure 4a) are noted over the blocks. 

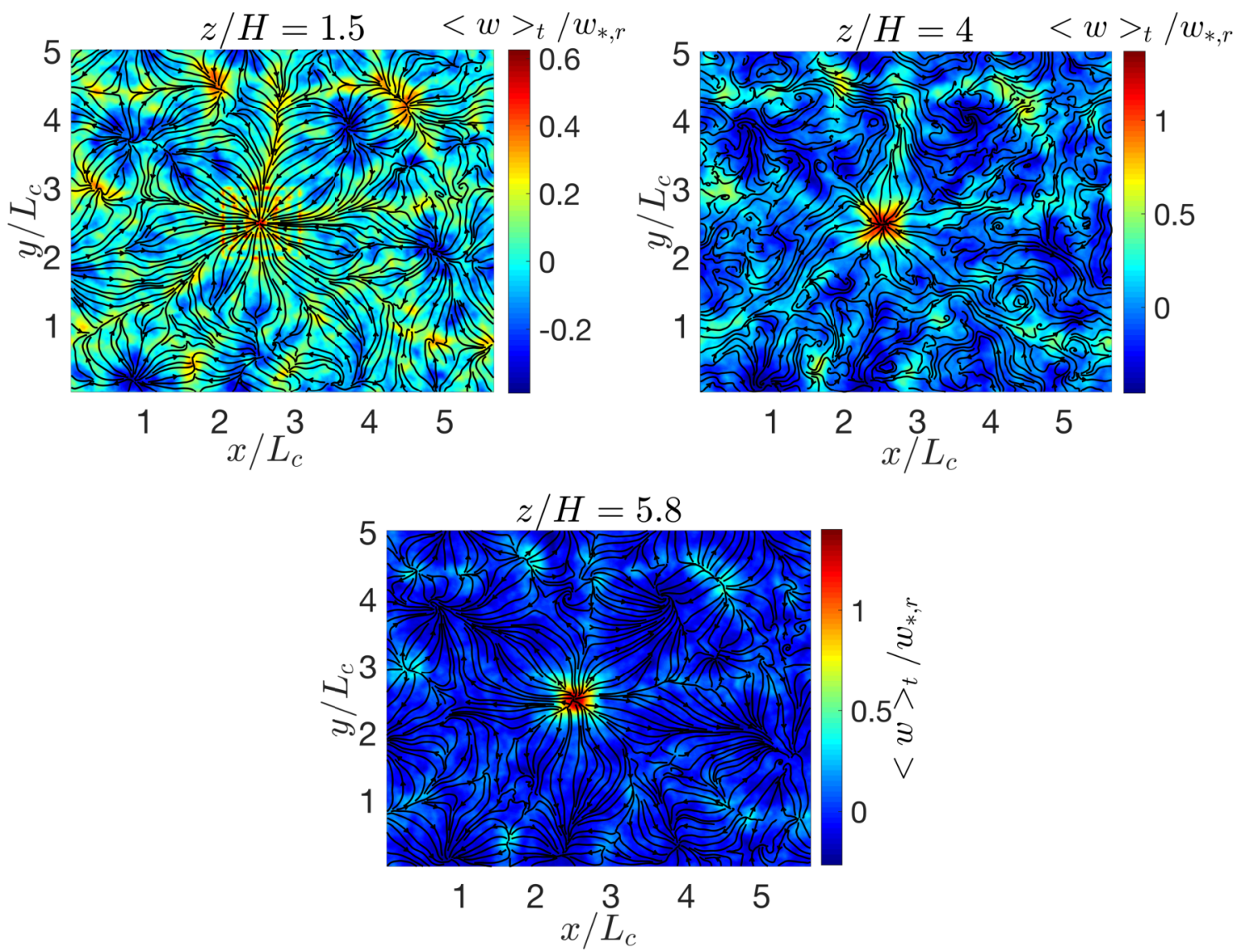

Figure 4. Pseudocolor plots the normalized time-averaged vertical velocity $\langle w\rangle$ over $x-y$ slices, for

336 the bubble regime (case 7), plotted for three different heights $\left(1.5 H\left(z / L_{c}=0.09\right), 4 H\left(z / L_{c}=0.23\right)\right.$, and $\left.3375.8 H\left(z / L_{c}=0.34\right)\right)$. The velocity is normalized by the convective velocity of rural area $\left(w_{*, r}\right)$. The lines

are the streamlines of the horizontal velocity.

\subsection{Spatial and time averaging}

Given the spatial heterogeneity of the flow, Reynolds averaging can only be surrogated for

341 by time averaging and we define perturbations only relative to a time average. Nevertheless, we

342 can also spatially-average any turbulent statistics over the city to identify flow structures or for

343 other analyses. For a variable $\varphi$, the averaged value is denoted as $\langle\varphi\rangle$, with subscripts to indicate 
344 the averaging dimensions. For example, $\langle\varphi\rangle_{t}$ is the Reynolds average, while $\langle\varphi\rangle_{y, z, t}$ means $\varphi$ is

345 averaged over $y$ and $z$, as well as temporally (but not in the $x$ direction). However, averaging in $x$ 346 and $y$ all through the paper is only done over the extent of the city in these dimensions, as depicted

347 in Figure 5. For the results shown in the $x-z$ plane, variables are averaged in the $y$ direction only 348 over the cross-stream span of the city (the buffer area is excluded from the averaging and analyses).

349 Similarly, for results shown in $y-z$ plane, averaging in the $x$ direction is over the streamwise span 350 of the city. In $z$, the variables are vertically averaged from top of the buildings up to $0.75 L_{z}$ to make 351 sure that the inversion (which starts at 0.8 of $L_{z}$ ) is excluded and its effects are minimized. For all 352 results shown in $x-z$ or $y-z$ planes, or $z$-profiles, the inversion is excluded. In addition, the volume 353 containing the buildings is not included in the averaging of the results or in the pseudocolor plots 354 in order to clearly illustrate the city location and the large-scale circulations around city.
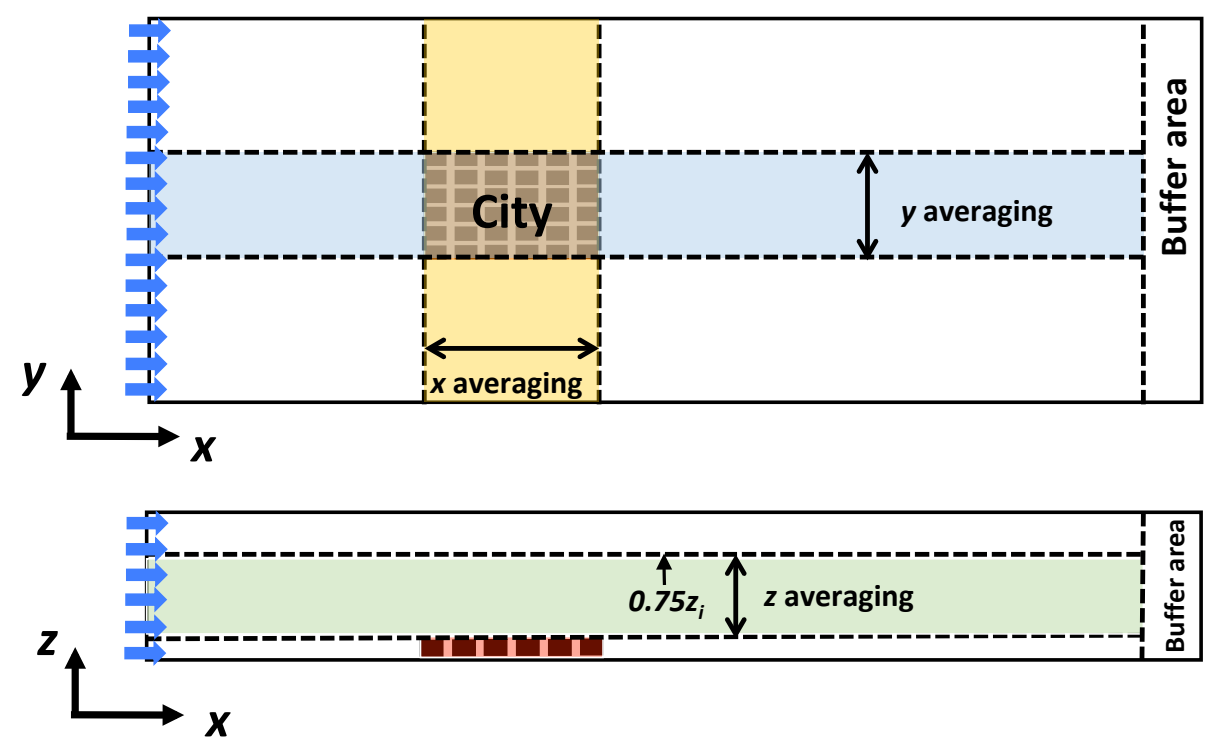

Figure 5. Schematic of the spatial averaging in $x$ and $y$ (top) and $z$ (bottom) directions

The turbulence statistics in all the simulations in Table 1 reach a statistically steady-state after an initial warm-up periods of about $16.8 \tau_{e}$, where $\tau_{e}$ is the large eddy turnover time defined 
here as $\tau_{e}=\frac{z_{i}}{\max \left(M, w_{*, u}\right)}$. This definition of eddy turnover time is consistent with the two non-

360 dimensional parameters derived in section $2\left(M / w_{*, r}\right.$ and $\left.w_{*, u} / w_{*, r}\right)$, and how the circulation

361 regime is hypothesized to depend on their relative magnitude. Statistical convergence analyses

362 based on the turbulent kinetic energy (TKE) profiles indicate that, after warm up is completed, 363 averaging over a time period of $22 \tau_{e}$ is sufficient.

\section{$364 \quad$ 4.3 Mixed advection-convection cases}

Figure 6 shows the pseudocolor and streamwise profiles of vertical velocity for cases 1 to

4. In all of the cases in this regime, both convection and advection are expected to be important

367 since $M / w_{*, r} \sim w_{*, u} / w_{*, r}$. Among these cases, case 2 is expected to be similar to the base case 1

368 since they have the same $M / w_{*, r}$ and $w_{*, u} / w_{*, r}$; indeed, their vertical velocities are quite similar

369 (they are not exactly identical probably due to inflow renormalization or incomplete statistical

370 convergence, but the differences are too small to be consequential so we did not probe this point

371 further). However, one can note that case 3 has stronger convective velocity over the city than the

372 base case since the ratio of $w_{*, u} / w_{*, r}$ for case 3 is higher than the base value (and heating of air

373 parcels near the surface is stronger). On the other hand, case 4 has a larger $M / w_{*, r}$ than the base

374 case, and we can observe from Figure 6e that for this case, the vertical velocity over the city is

375 weaker than in the base case. From figures 6 a-d, patches of higher vertical velocity are observed

376 immediately above the city block; they are due to the combination of upward deflection of the

377 mean flow as it impinges on the buildings and uplift inside the city streets as the streamwise flow 378 decelerates and heats up and the air rises. 
Figure 7 a-d show the $u$ velocity map, and Figure 7 e shows the vertical profile over the

380 city for cases 1 to 4 . In general, for all of these cases, $u$ has a peak over the city, and decreases near

381 the top of ABL. This peak in the $u$ velocity is mainly due to stronger buoyant mixing that

382 homogenizes the $u$ profile throughout the domain such that the acceleration due to flow deflection

383 above the city is relatively more significant and becomes a peak in the profiles. Figure 7 e indicates

384 that, while $u$ in case 2 agrees well with the one for the base case as expected, in case 3 it slows

385 down at the top of ABL relative to the base case 1. Finally, case 4 has a higher $u$ velocity than the

386 base case due to a larger ratio of $M / w_{*, r}$, resulting in a lower $w$ and a higher $u$ (for comparison

387 of the horizontal profile of TKE, the reader is referred to Appendix D). These results again support

388 our dimensionless scaling of the problem.
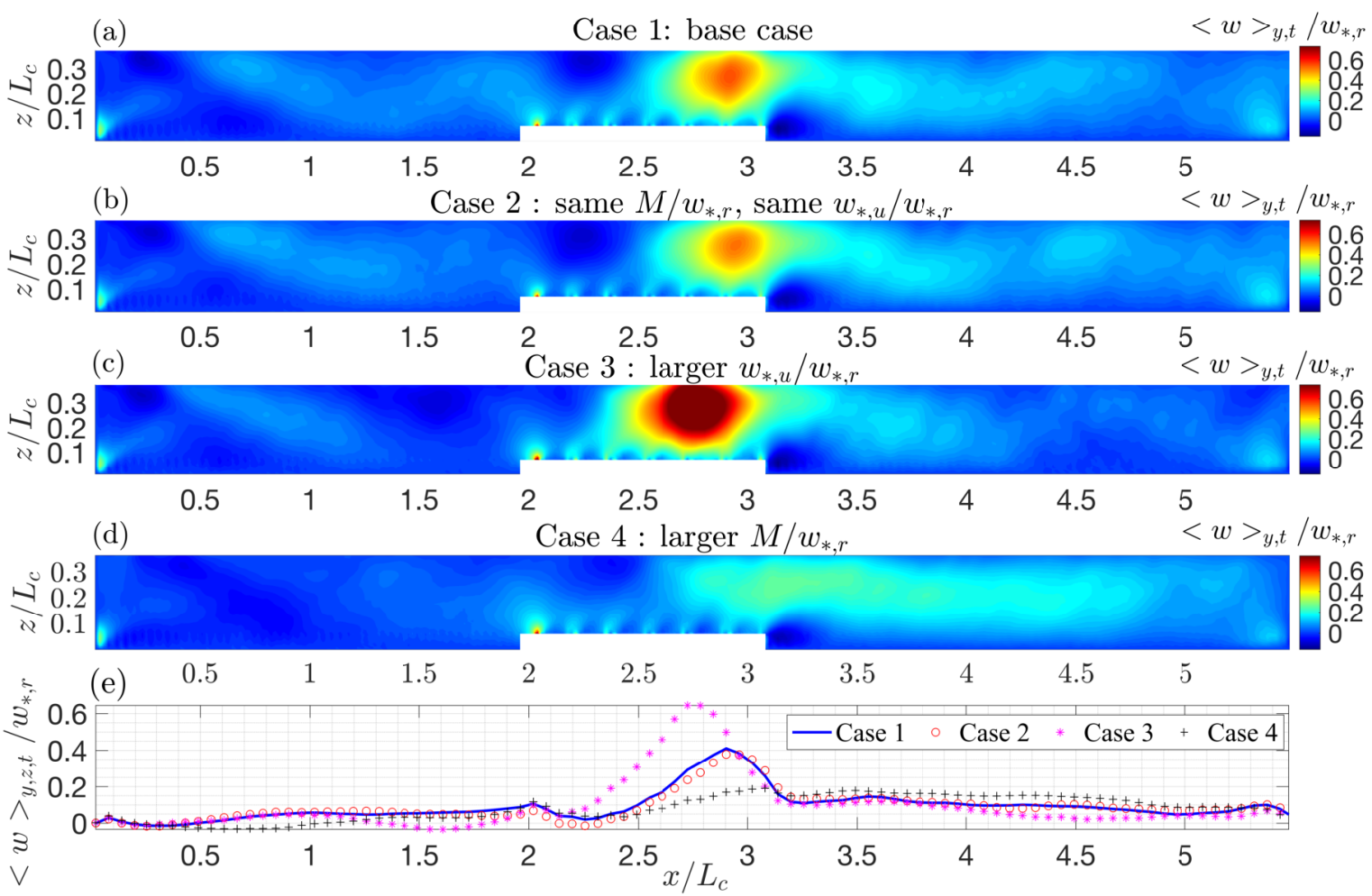

(a)
Case 1: base case
$<u>_{y, t} / w_{*, r}$

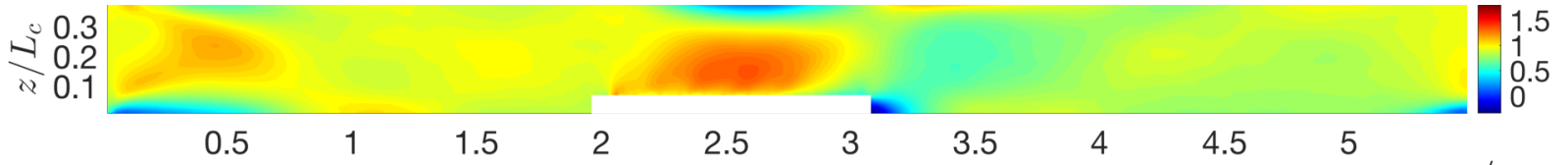

(b) Case 2 : same $M / w_{*, r}$, same $w_{*, u} / w_{*, r} \quad<u>_{y, t} / w_{*, r}$

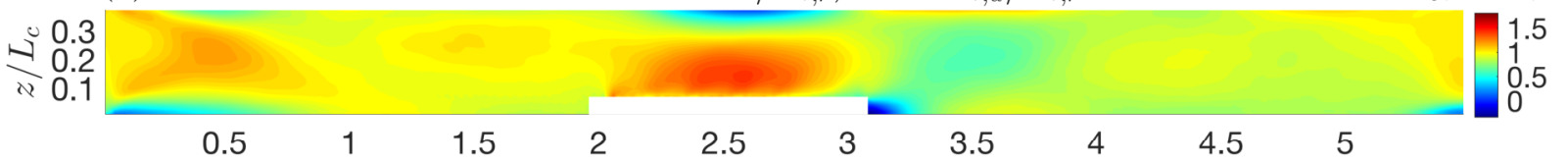

(c) $\quad$ Case 3: larger $w_{*, u} / w_{*, r} \quad<u>_{y, t} / w_{*, r}$

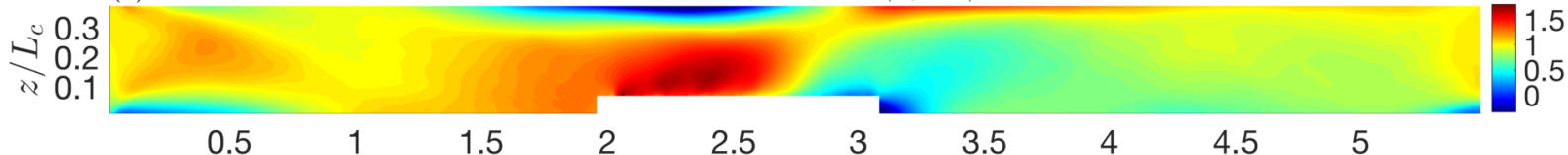

(d) Case 4: larger $M / w_{*, r} \quad<u>_{y, t} / w_{*, r}$
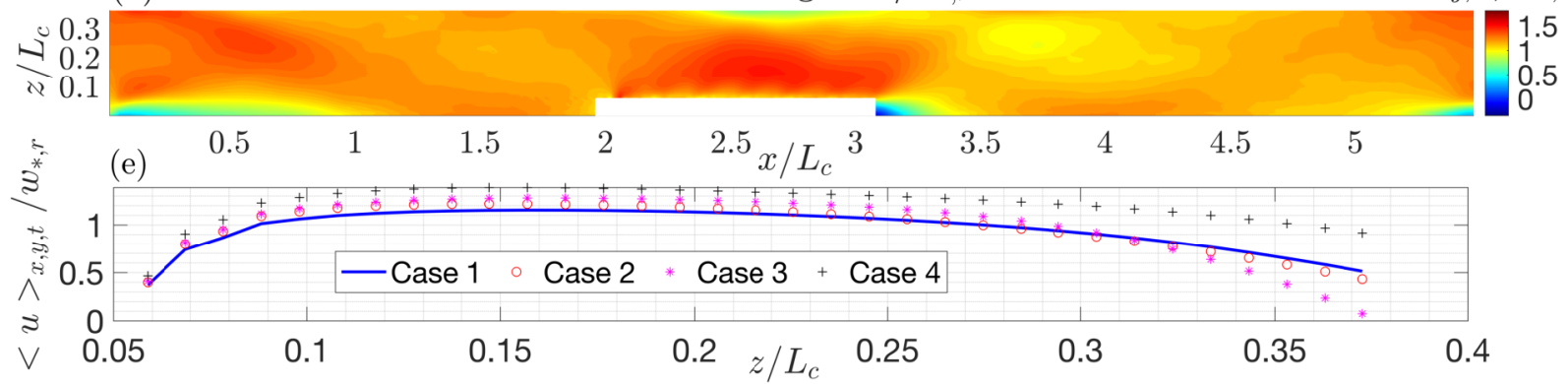

Figure 7. Pseudocolor plots of $u$ (normalized by $w^{*}, r$ ) for cases 1 (a), 2 (b), 3 (c) and 4 (d), and vertical profile of $u$ (normalized by $w_{*, r}$ ) over the city for case 1 to 4 (e).

\subsection{Advection dominated cases}

Figure $8 \mathrm{a}$ and $\mathrm{b}$ show the pseudocolor plots of vertical velocity for Cases 5 and 6 . In these cases, we used $w^{*}, r$ for normalization to be consistent with equation (2); however, $M$ can also be used for normalization (it would be more physically informative), and it leads to similar but scaled

400 plots since these two cases have similar $M$ and $w^{*}, r$. One can note that, although these two cases

401 have different $w_{*, u} / w_{*, r}$, they have similar vertical velocity $(w)$ contours, and horizontal profiles

402 (Figure 8c) since for these two cases, $M / w_{*, r} \gg w_{*, u} / w_{*, r}$. These results confirm that the only 
403 important parameter in this limit is $M / w_{*, r}$. One can reach a similar conclusion by examining the 404 pseudocolor plots of the streamwise velocity $(u)$, and its vertical profile in Figure 9. Cases 5 and 6 405 are associated with the advection-dominated regimes where the flow is modulated by the inflow, 406 with no noticeable thermal buoyancy impacts. In these cases, the location of the largest vertical 407 velocity is just upstream of the city where the inflow first experiences the blockage impact of the 408 roughness elements of the city. In addition, downstream of the city, a recirculation zone is observed 409 with negative $w$ and $u$ values (for comparison of the horizontal profile of TKE, the reader is 410 referred to Appendix E).

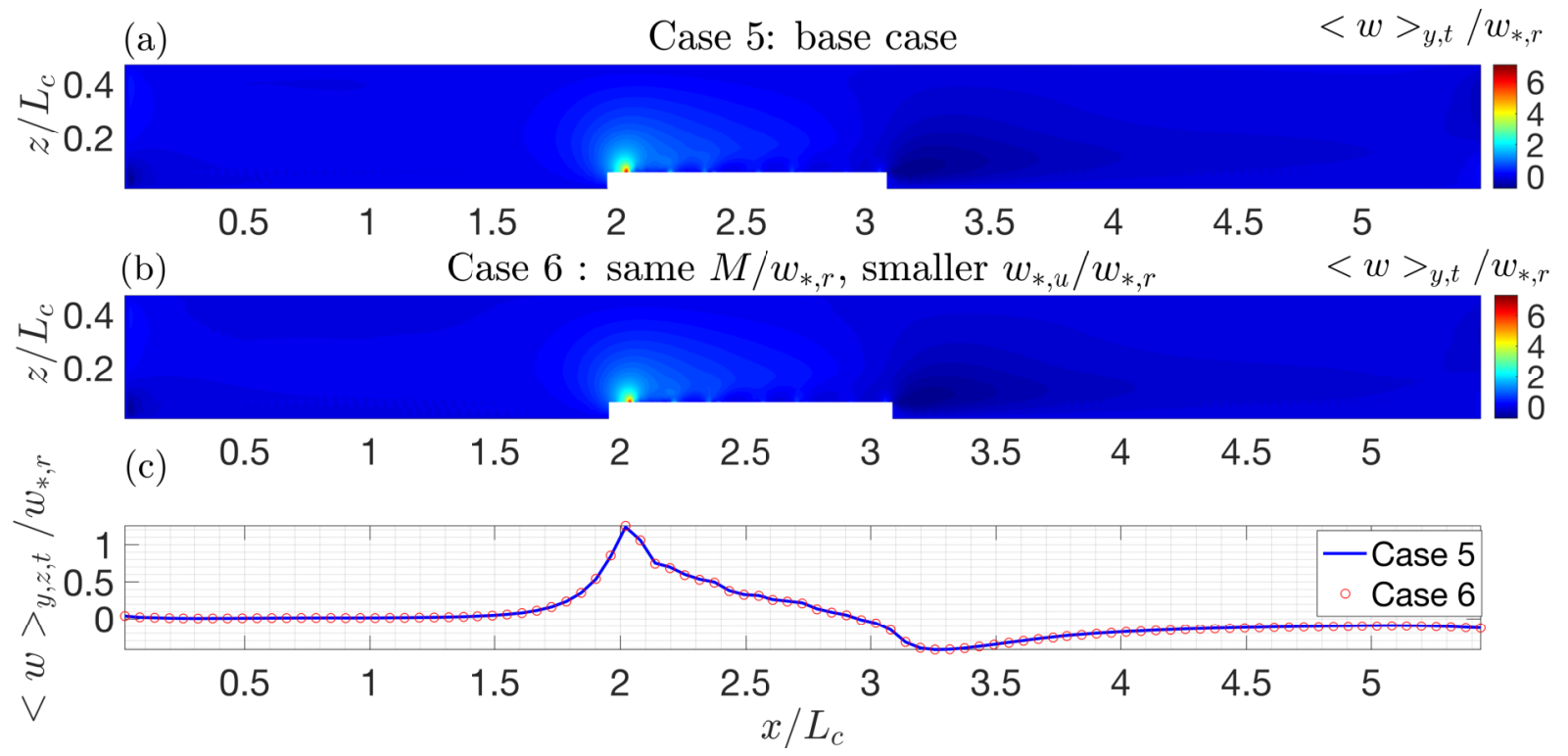
Figure 8. Pseudocolor plots of normalized (normalized by $w_{*, r}$ ) $w$ for cases 5 (a) and 6 (b), and streamwise profile of $w$ for these two cases (c). 


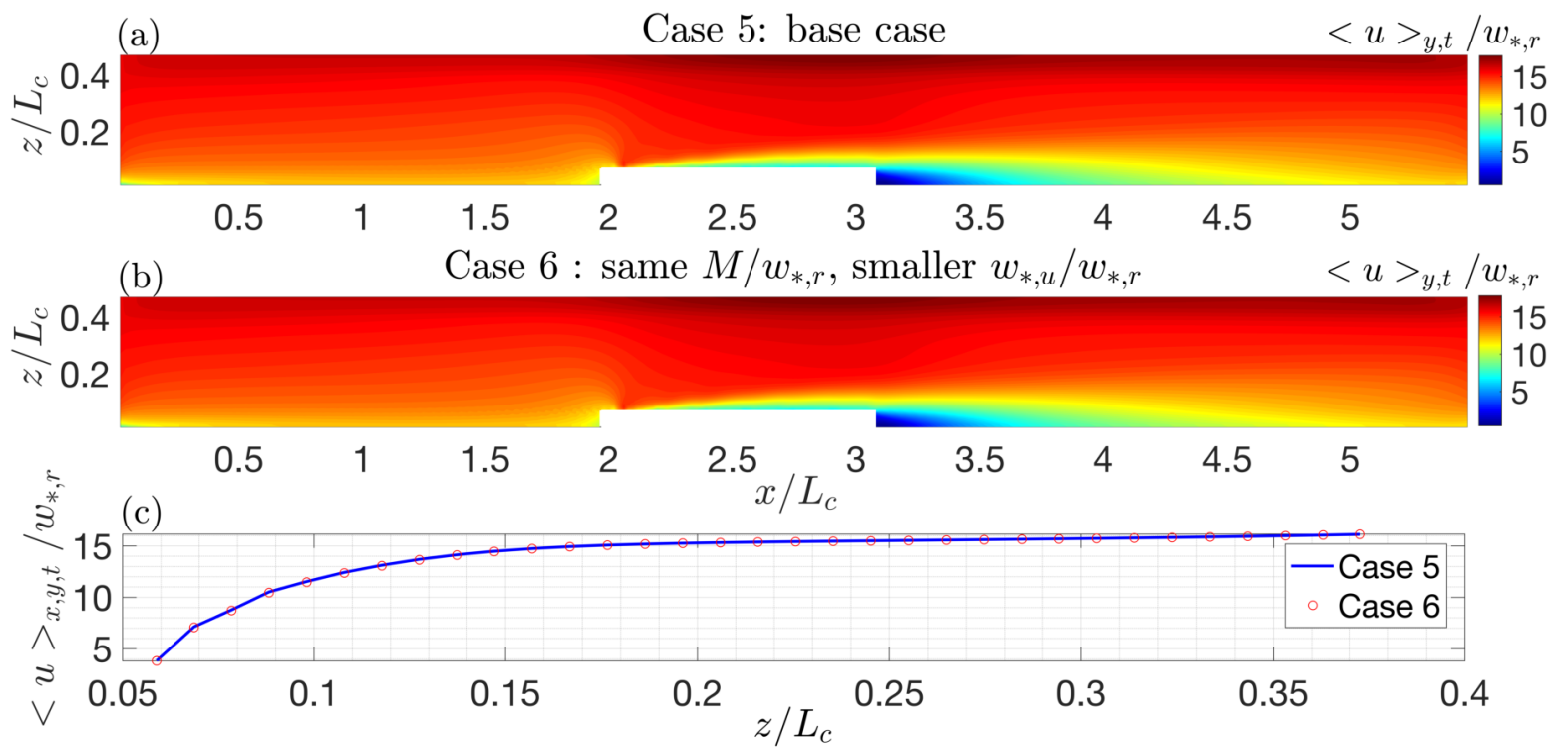

414

Figure 9. Pseudocolor plots of normalized (normalized by $w_{*, r}$ ) $u$ for cases 5 (a) and 6 (b), and vertical profile of $u$ over the city for these two cases (c).

\section{$417 \quad 4.5$ Convection dominated cases}

In cases 7 and 8, convection is the main driver of the circulation around the city. In these

419 cases, the buoyancy force lifts parcels of air from the city while the advective wind is too weak to

420 move these parcels away from the city. Therefore, the convective updraft rises to the top of ABL

421 where it meets the inversion and diverges outwards. Then, surface level convergence occurs, and

422 the thermal circulation is completed by a downdraft around the city that results in a bubble. Figure

42310 shows the maps and the horizontal profile of the vertical velocity for cases 7 and 8 . As can be

424 noted for these cases, the vertical velocity peaks over the city. In addition, since the ratio $w_{*, u} / w_{*, r}$

425 is the same for both cases, their normalized vertical velocities match despite the facts that (i) the

426 values of $w_{*}$ for the urban and rural areas are both different for the two cases and (ii) $M / w_{*, r}$ for

427 cases 8 is larger than case 7 . Figure 11 shows the $u$ velocity for cases 7 and 8 , depicting two 428 identical large circulations that extend upstream and downstream of the city. These two 
429 circulations are separated in the middle of the city as can be observed from Figure 11c that shows

430 the vertical profile of $u$ over each half of the city (right and left sides relative to inflow direction).

431 In general, the similarity of the results of cases 7 and 8 verify our scaling arguments that in the

432 limit of $M / w_{*, r} \ll w_{*, u} / w_{*, r}$, the only important ratio is $w_{*, u} / w_{*, r}$ (for comparison of the

433 horizontal profile of TKE, the reader is referred to Appendix E)..

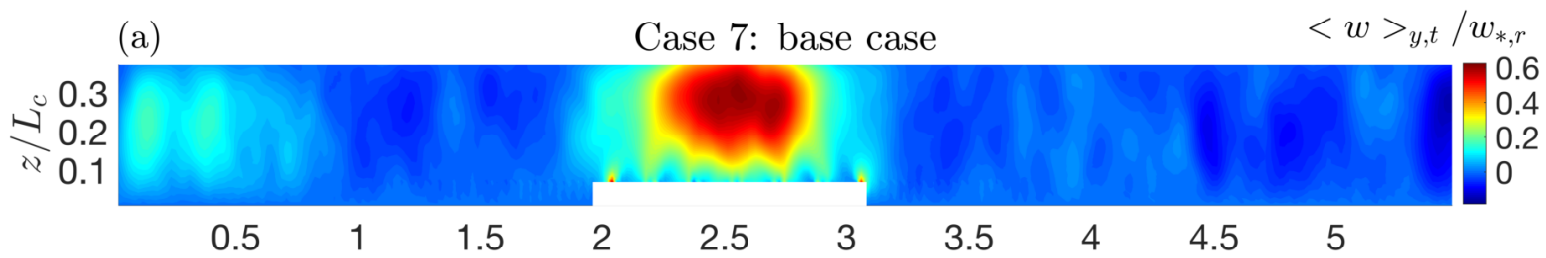

(b) Case 8 : larger $M / w_{*, r}$, same $w_{*, u} / w_{*, r} \quad<w>_{y, t} / w_{*, r}$

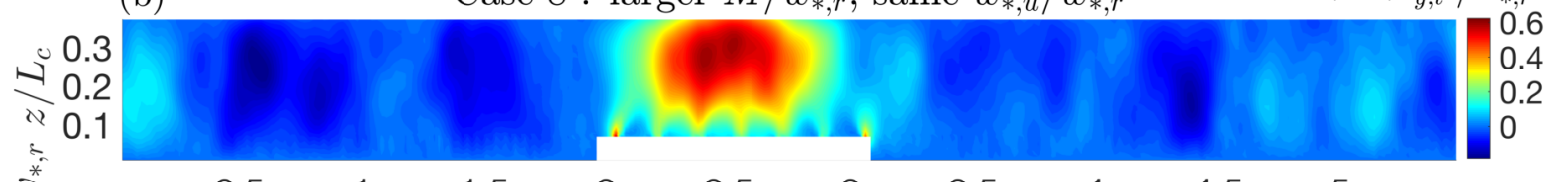

434

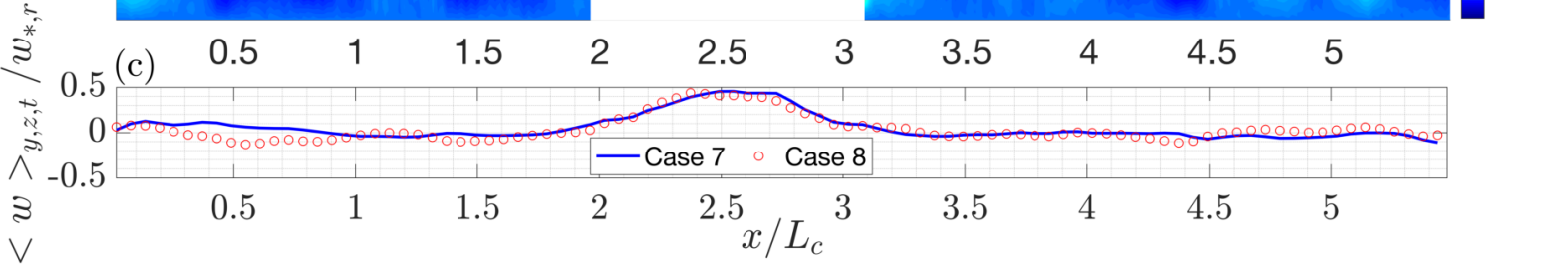

Figure 10. Pseudocolor plots of normalized $w$ for cases 7 (a) and 8 (b), and streamwise profile of 


\section{(a)}

Case 7: base case

$<u>_{y, t} / w_{*, r}$

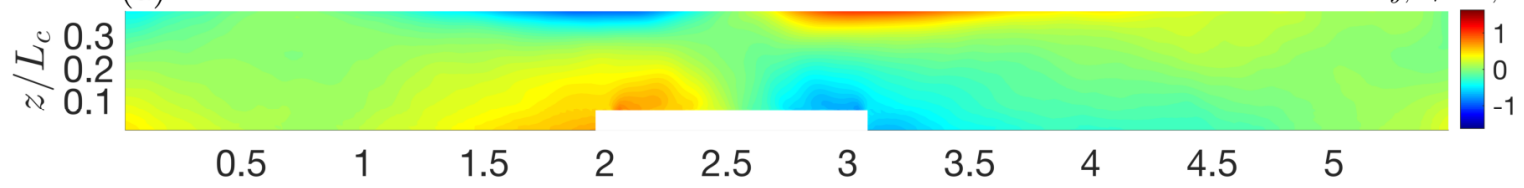

(b)

Case 8 : larger $M / w_{*, r}$, same $w_{*, u} / w_{*, r}$

$<u\rangle_{y, t} / w_{*, r}$
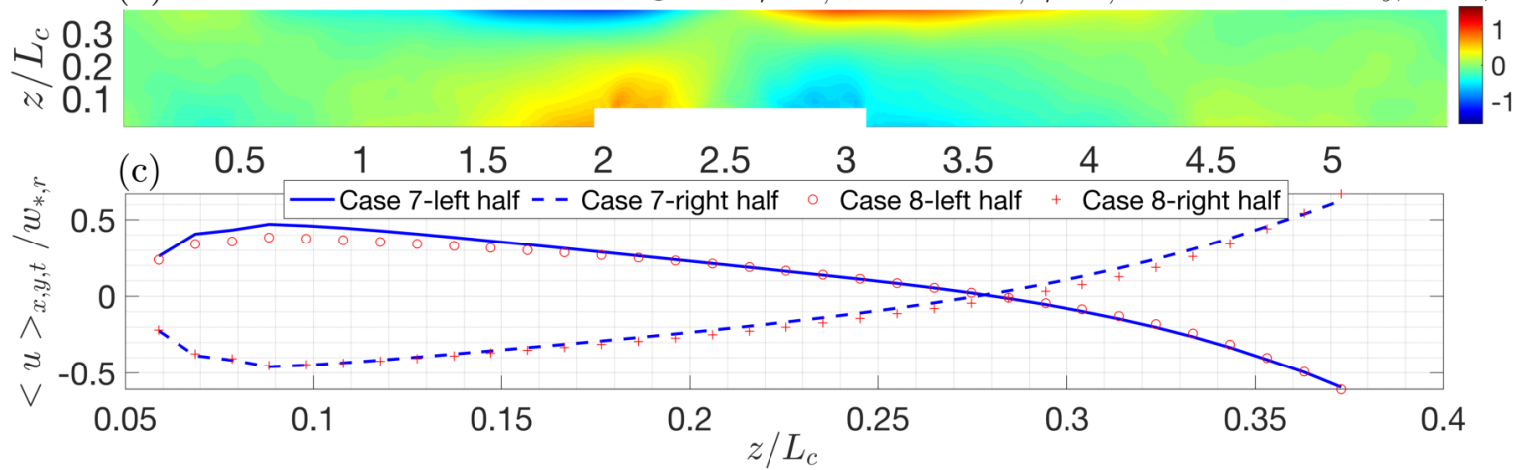

Figure 11. Pseudocolor plots of normalized (by $w_{*, r}$ ) $u$ for cases 7 (a) and 8 (b), and vertical profile of $u$ over the left and right side of the city for these two cases (c).

\section{Large scale circulation: plume to bubble transition}

In previous sections, we demonstrated that the circulations around the city can be scaled

442 with two non-dimensional parameters: $w_{*, u} / w_{*, r}$ and $M / w_{*, r}$. Now, using these two non-

443 dimensional numbers, we investigate how and where the circulations around the city transition

444 from a fully advection dominated regime (plume) to a fully convection dominated one (bubble),

445 and what lies in the intermediate transition region. To that end, we conduct a larger suite of lower-

446 resolution simulations with different values $w_{*, u} / w_{*, r}$ and $\frac{M / w_{*, r}}{w_{*, u} / w_{*, r}}=M / w_{*, u}$. Note that the

447 second parameter is the ratio of the two dimensionless parameters that we derived previously, and

448 as such it is itself a dimensionless parameter that can be used along with only one of the other two

449 to describe the dynamics (non-dimenional parameter sets are not unique). We select it here since

450 it more conveniently delimits the parameter space we need to cover, as shown in Figure 12. Since 
451 we are mostly interested in the general behavior of the circulations, and to cover the largest span

452 of the parameter space with the computational resource available, these simulations are conducted

453 at half the resolutions of the main cases in Table 1 (with number of grid points in $x, y$ and $z$ equal

454 to 144,128 , and 24 respectively). To ensure that the effect of the resolution is insignificant on the

455 large circulations we are examining, we perform direct flow comparison and grid sensitivity

456 analysis in Appendix 1, and the conclusions from these low-resolution simulations are later

457 verified using the high-resolution cases in Table 1. A total of 66 simulation are performed with

$458 \quad 1.4 \leq w_{*, u} / w_{*, r} \leq 4$ and $0.3 \leq M / w_{*, u} \leq 3$. Table 2 shows the parameters of these simulations.

Table 2. Parameters of the low-resolution simulations: we use six values of $w_{*, u} / w_{*, r}$, and for each of these we simulate 11 values of $M / w_{*, u}$, resulting in a total of 66 simulations spanning all the possible combinations of the two non-dimensional parameters.

\begin{tabular}{|c|c|}
\hline$\frac{w_{*, u}}{w_{*, r}}$ & $\frac{M}{w_{*, u}}$ \\
\hline $1.4,1.8,2.5,3,3.5,4$ & $0.3,0.4,0.5,0.6,0.8,1,1.4,1.8,2.2,2.6,3$ \\
\hline
\end{tabular}

Parameter space for low-resolution cases

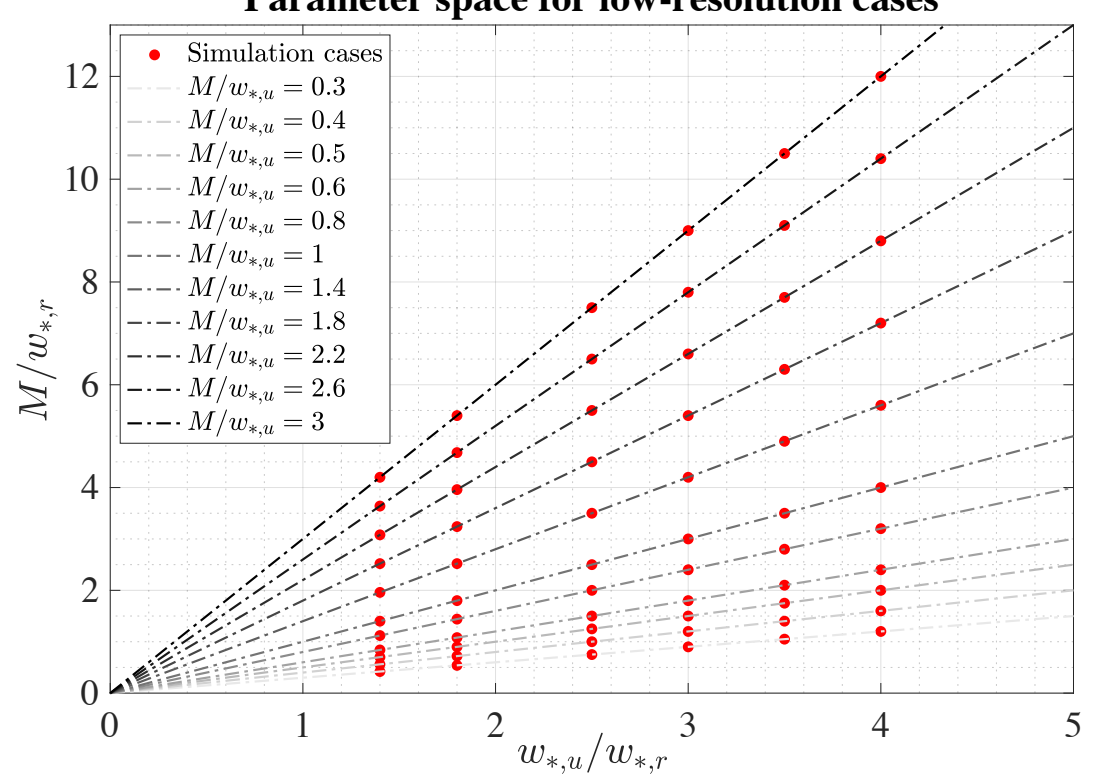




\subsection{Criteria for plume, bubble and transition regimes}

To define the proper criteria for categorizing different circulation regimes as bubble, plume, or transitional, we consider the streamwise evolution of $w$ (averaged temporally and also

467 spatially in the $y$ and $z$ directions over the city). Figure 13 shows this profile when $w_{*, u} / w_{*, r}=3$

468 and for different $M / w_{*, u}$ values from Table 2. From this figure, we can observe the following:

469 i) For low $M / w_{*, u}=0.3,0.4,0.5,0.6, w$ has a peak over the city. The location of this peak is

470 in the middle of the city for smaller $M / w_{*, u}$ values in that range, and while it shifts downstream

471 for stronger inflow velocity, it remains over the city. For these cases generally, the profile of $w$ 472 downstream of the city is negative. Here, using a visual inspection of the flow fields of these cases, 473 we classify them into a bubble regime.

474 ii) For high $M / w_{*, u}=1.8,2.2,2.6,3, w$ has a peak upstream of the city where the inflow first 475 meets the city and is diverted upwards. Then, due to the flow recirculation, it becomes negative 476 downstream where the flow subsides due to continuity and streamwise acceleration (as reported in 477 Bou-Zeid et al. (2009)) . For these cases generally, the averaged value of the streamwise gradient 478 of $w$ downstream of the city is positive. These cases are classified as belonging to the plume 479 regime.

480 iii) For intermediate values of $M / w_{*, u}=0.8,1,1.4$, there are two main peaks in $w$ profile. One 481 is associated with the advective upward deflection at the upstream edge of the city, and another is 482 related to the convective updrafts at the downstream end, but still over, the city. For these cases, 483 the averaged value of the streamwise gradients of $w$ downstream of the city switches between 484 positive and negative. We consider these cases as transitional regimes that are intermediate 485 between plumes and bubbles since they display features from both types. 


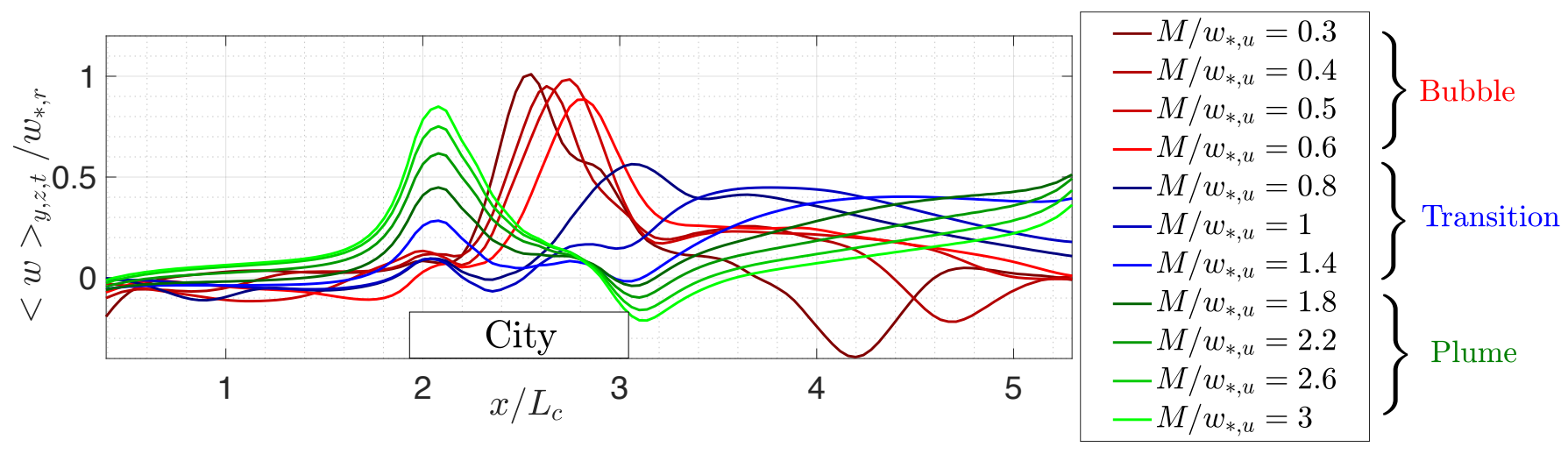

Figure 13. Streamwise profiles of $\langle w\rangle_{y, z, t}$ for the case with $w_{*, u} / w_{*, r}=3$, and for different $M / w_{*, u}$ based on Table 2.

Using the above characteristics of each regime, we are now able to distinguish the cases in

490 Table 2, and bin them into the three regimes. Figure $14 \mathrm{a}$ shows the categorization of all

491 simulations. It can be observed from this figure that depending on the ratio of two non-dimensional

492 parameters, $\frac{M / w_{*, r}}{w_{*, u} / w_{*, r}}=\frac{M}{w_{*, u}}$, we are able to classify the cases as plume $\left(M / w_{*, u}>1.7\right)$,

493 transitional $\left(0.7 \leq M / w_{*, u} \leq 1.7\right)$, and bubble $\left(M / w_{*, u}<0.7\right)$ regimes. In addition, Figure $14 \mathrm{~b}$

494 shows that the classification of the high-resolution cases in Table 1, based on the same criterion,

495 matches the results of the low-resolution cases. This confirms that the large circulations types are

496 insensitive to the resolution in the range of resolutions we use. We note that while this finding

497 indicates that only one non-dimensional parameter is needed to classify the flow regime,

498 significant changes may still be noted within each regime as the other non-dimensional velocity

499 ratio varies. 

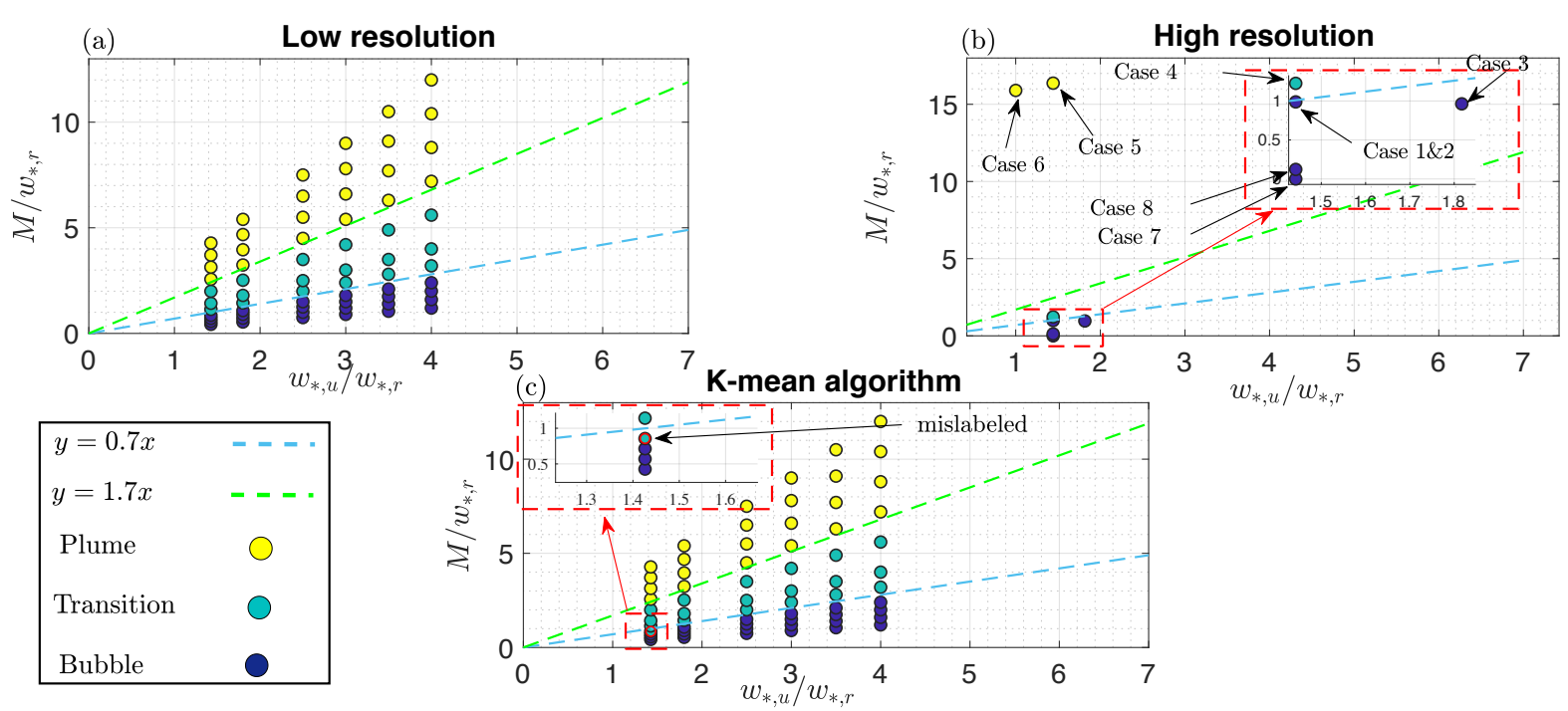

500 clustering algorithm (Lloyd 1982), is provided with streamwise profiles of $w$ for all cases in Table 2 as vectors with $n_{x}$ elements ( $=144$ in this case, the number of grid points along $x$ ) elements, and the desired number of clusters (=3 in this case) is imposed (kmeans MATLAB function is used

510 for this purpose: MathWorks 2019). The algorithm tries to cluster all profiles based on their 511 extracted characteristics without any intervention by the user. Figure 14c shows the results using 512 the k-means clustering algorithm, which classifies each data point to the cluster with closest mean 513 to that data point and thus minimizes the variance between the members within each of the clusters. 514 Overall, the algorithm clusters almost all the cases exactly as in our "visual expert classification" 
515 in Figure 14a, except for one case that is very close to the border of plume-transition cases. This

516 indeed confirms that the transition criterion postulated above holds broadly.

\section{Conclusion and implications}

Mixed convection heat transfer is an important process in various applications and at

519 various scales. A particularly relevant geophysical application concerns the heat exchange between

520 the atmospheric boundary layer and urban areas (which are hotter than their surroundings due to

521 the urban heat island effect); the resulting flow patterns affect the air quality and temperature in

522 cities. In this paper, we used LES to study city-scale circulations, and how their dynamics are

523 jointly modulated by the wind speed and the heat flux of urban and rural areas.

$524 \quad$ Using dimensional analysis and keeping the geometry related parameters fixed for this

525 study, two parameters are shown to govern the behaviour of circulations above cities: (1) the ratio

526 of the convective velocity of urban area over that of the rural area, and (2) the ratio of the

527 bulk/average inflow velocity over the convective velocity of the rural area. Depending on the

528 relative magnitude of these two dimensionless parameters, city-scale circulations change from

529 natural/pure convective driven circulations, where the first ratio is the only important one, to

530 advection dominated circulations, where the second ratio solely controls dynamics of circulations.

531 An intermediate regime exists where both ratios are important, and ABL circulations are driven

532 by both advection and convection processes (mixed convection). In addition, using the horizontal

533 transects of the vertical velocity, we proposed a single a priori (based on inputs) criterion to

534 classify the different city-scale circulations (with different dimensionless parameters) into three

535 regimes: bubble, transition, and plume. The classification was then confirmed using blind k-means

536 clustering. While in this paper, we only focused on the influence of urban/rural heat flux and bulk 
537 velocity of the flow, future studies are encouraged to investigate the effect of geometry related 538 parameters that were fixed in our study, such as city size and surface roughness.

539 The implication of this work for city ventilation, and how it is influenced by ABL-scale 540 circulations, are myriad. We can already make some conclusions regarding the effect of the flow 541 regime on the thermal environment in the city, as illustrated in Figure 15. The figure contrasts the 542 temperature pseudocolor and velocity streamlines in the $x-z$ plane for different circulation regimes.

543 The cases are from the lower resolution runs and for a constant $w^{*}, u / w^{*}, r=3$. One can note that for 544 low $M / w_{*}, r$ (bubble cases, minimum ventilation), the air above the city is hotter than at larger $545 M / w^{*}, r$, and the heat generated in the city is lofted vertically above the city and recirculated back 546 to the city. On the other hand, in the case of a plume regime (maximum ventilation), the heat is 547 transported mostly downstream of the city (and leaves the domain) leading to a lower temperature 548 in the city and a lower maximum temperature over the whole domain. The transitional regimes are 549 associated with partially ventilated conditions. While these application-specific impacts will be 550 more closely examined in follow-up studies, this paper lays the dimensional analysis and scaling 551 grounds on which these subsequent studies can build. 

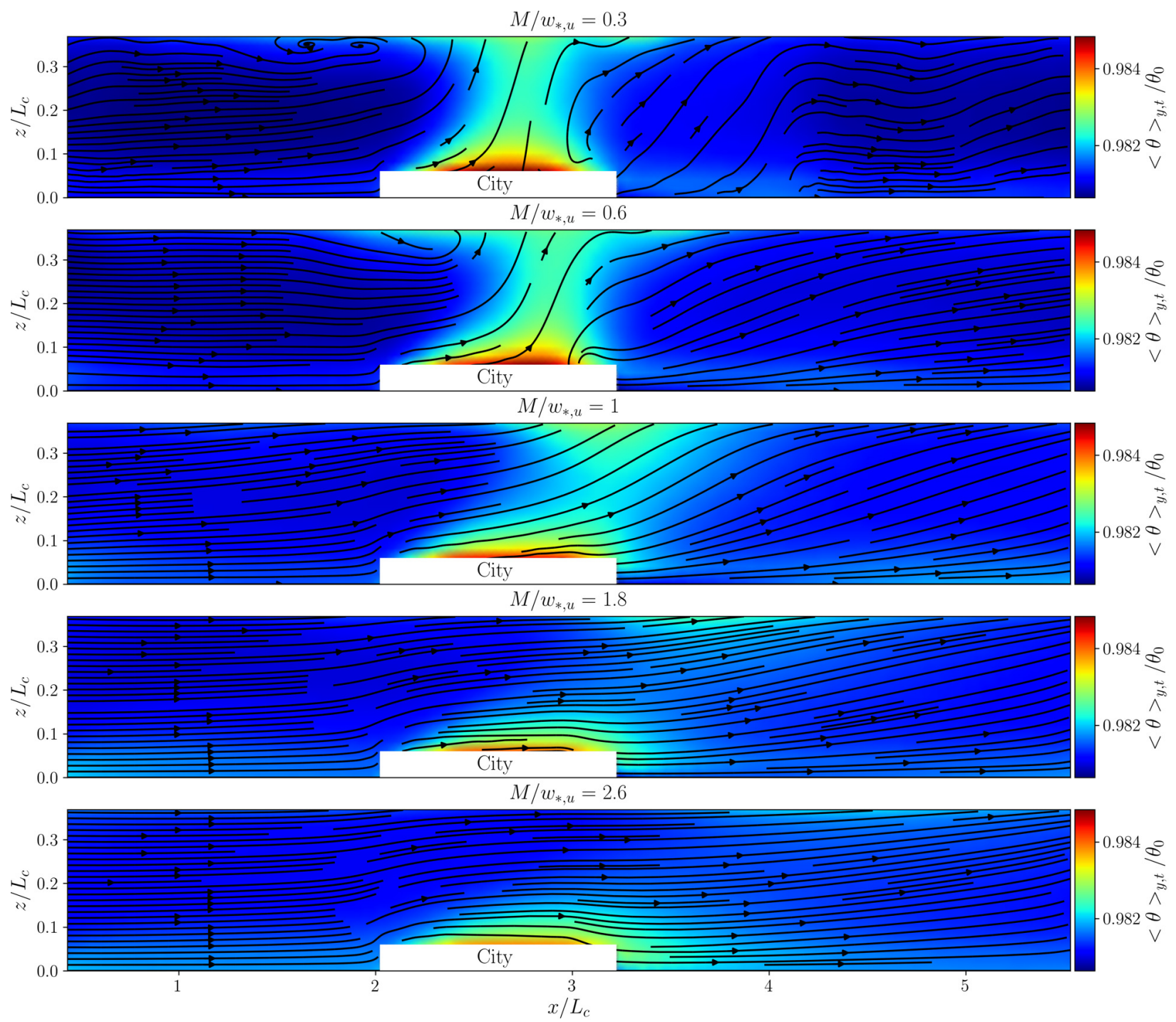

Figure 15. Pseudocolor plots of temperature (normalized by reference temperature) and velocity streamline in $z-x$ plane for different $M / w_{*, r}$ but constant $w_{*, u} / w_{*, r}=3$ (low resolution simulations as discussed in section 5.1). The white masked area contains both city blocks and streets (fluids space).

\section{Acknowledgement}


ICER1664091 and CBET1444758 (The Urban Water Innovation Network SRN). The simulations

562 are conducted on the Cheyenne supercomputer of the National Center for Atmospheric Research

563 under project numbers UPRI0007 and UPRI00016.

564

565 Declaration of interest: The Authors report no conflict of interest

\section{Reference}

567 Ahlers, G., Grossmann, S., \& Lohse, D. (2009). Heat transfer and large scale dynamics in turbulent 568 Rayleigh-Bénard convection. Reviews of Modern Physics, 81(2), 503-537.

569 Akbarinia, A., \& Behzadmehr, A. (2007). Numerical study of laminar mixed convection of a 570 nanofluid in horizontal curved tubes, 27, 1327-1337.

571 Bejan, A. (1993). Heat transfer, New York, NY: John Wiley \& Sons, Inc.

572 Bergman, T. L., Incropera, F. P., DeWitt, D. P., \& Lavine, A. S. (2011). Fundamentals of heat and 573 mass transfer, John Wiley \& Sons.

574 Bou-Zeid, E., Meneveau, C., \& Parlange, M. (2005). A scale-dependent Lagrangian dynamic 575 model for large eddy simulation of complex turbulent flows. Physics of Fluids, 17(2), 1-18.

576 Bou-Zeid, E., Meneveau, C., \& Parlange, M. B. (2004). Large-eddy simulation of neutral 577 atmospheric boundary layer flow over heterogeneous surfaces: Blending height and effective 578 surface roughness. Water Resources Research, 40(2). doi:10.1029/2003WR002475

579 Bou-Zeid, E., Overney, J., Rogers, B. D., \& Parlange, M. B. (2009). The Effects of Building 580 Representation and Clustering in Large-Eddy Simulations of Flows in Urban Canopies. 581

582 Changnon, S. A. (1979). Rainfall changes in summer caused by St. Louis. Science, 205(4404), 583 402-404. 
De Foy, B., Varela, J. R., Molina, L. T., \& Molina, M. J. (2006). Rapid ventilation of the Mexico City basin and regional fate of the urban plume. Atmospheric Chemistry and Physics, 6(8), $2321-2335$.

Deardorff, J. W. (1970). Convective velocity and temperature scales for the unstable planetary boundary layer and for Rayleigh convection. Journal of the Atmospheric Sciences, 27(8), $1211-1213$.

Fan, Y., Li, Y., Bejan, A., Wang, Y., \& Yang, X. (2017). Horizontal extent of the urban heat dome flow. Scientific Reports, 7(1), 1-10.

Fan, Y., Li, Y., Wang, X., \& Catalano, F. (2016). A new convective velocity scale for studying diurnal urban heat island circulation. Journal of Applied Meteorology and Climatology, 55(10), 2151-2164.

Fan, Y., Li, Y., \& Yin, S. (2018). Non-uniform ground-level wind patterns in a heat dome over a uniformly heated non-circular city. International Journal of Heat and Mass Transfer, 124, $233-246$.

Hataya, N., Mochida, A., \& Iwata, T. (2014). Development of the simulation method for thermal environment and pollutant diffusion in street canyons with subgrid scale obstacles, (2), 553556.

Huang, J., \& Bou-Zeid, E. (2013). Turbulence and Vertical Fluxes in the Stable Atmospheric Boundary Layer. Part I: A Large-Eddy Simulation Study. Journal of the Atmospheric Sciences, 70(6), 1513-1527.

Kimura, R. (1976). Effects of General Flows on a Heat Island Convection. Journal of the Meteorological Society of Japan. Ser. II, 54(5), 308-320.

Klein, P. M. (2012). Metropolitan effects on atmospheric patterns: Important scales. In 
608 Klemp, J. B., \& Lilly, D. K. (1978). Numerical Simulation of Hydrostatic Mountain Waves. 609 Journal of the Atmospheric Sciences, 35(1), 78-107.

610 Kurbatskii, A. F., \& Kurbatskaya, L. I. (2016). Turbulent circulation above the surface heat source 611 in stably stratified atmosphere. AIP Conference Proceedings, 1770. doi:10.1063/1.4963976

612 Li, D. (2016). Revisiting the subgrid-scale Prandtl number for large-eddy simulation. Journal of $613 \quad$ Fluid Mechanics, 802, R2.

614 Li, Q., \& Bou-Zeid, E. (2019). Contrasts between momentum and scalar transport over very rough $615 \quad$ surfaces. Journal of Fluid Mechanics, 880, 32-58.

616 Li, Q., Bou-Zeid, E., \& Anderson, W. (2016a). The impact and treatment of the Gibbs phenomenon 617 in immersed boundary method simulations of momentum and scalar transport. Journal of $618 \quad$ Computational Physics, 310, 237-251.

619 Li, Q., Bou-Zeid, E., Anderson, W., Grimmond, S., \& Hultmark, M. (2016b). Quality and 620 reliability of LES of convective scalar transfer at high Reynolds numbers. International Journal of Heat and Mass Transfer, 102, 959-970.

Llaguno-Munitxa, M., \& Bou-Zeid, E. (2018). Shaping buildings to promote street ventilation: A large-eddy simulation study. Urban Climate, 26(May), 76-94.

Lloyd, S. P. (1982). Least Squares Quantization in PCM. IEEE Transactions on Information Theory, 28(2), 129-137.

Lund, T. S., Wu, X., \& Squires, K. D. (1998). Generation of Turbulent Inflow Data for SpatiallyDeveloping Boundary Layer Simulations. Journal of Computational Physics, 140(2), 233258.

MathWorks. (2019). MATLAB kmeans function. Retrieved January 25, 2020, from 
https://mathworks.com/help/stats/kmeans.html

Mochida, A., Tabata, Y., Iwata, T., \& Yoshino, H. (2008). Examining tree canopy models for CFD prediction of wind environment at pedestrian level. Journal of Wind Engineering and Industrial Aerodynamics, 96(10-11), 1667-1677.

Monin, A. S., \& Obukhov, A. M. (1954). Basic laws of turbulent mixing in the surface layer of the atmosphere. Contrib. Geophys. Inst. Acad. Sci. USSR, 24(151), 163-187.

Niino, H., Mori, A., Satomura, T., \& Akiba, S. (2006). Flow Regimes of Nonlinear Heat Island Circulation. Journal of the Atmospheric Sciences, 63(5), 1538-1547.

Oke, T. R. (1982). The energetic basis of the urban heat island. Quarterly Journal of the Royal Meteorological Society, 108(455), 1-24.

Peskin, C. S. (2002). The immersed boundary method. Acta Numerica, 11(2002), 479-517.

Petukhov, B. S., Polyakov, A. F., \& Launder, B. E. (1988). Heat transfer in turbulent mixed convection.

Ryu, Y. H., Baik, J. J., \& Han, J. Y. (2013). Daytime urban breeze circulation and its interaction with convective cells. Quarterly Journal of the Royal Meteorological Society, 139(671), 401413.

Sawai, T. (1978). Formation of the Urban Air Mass and the Associated Local Circulation. Journal of the Meteorological Society of Japan. Ser. II, 56(3), 159-174.

Shah, S., \& Bou-Zeid, E. (2014). Very-Large-Scale Motions in the Atmospheric Boundary Layer Educed by Snapshot Proper Orthogonal Decomposition. Boundary-Layer Meteorology, 153(3), 355-387.

Shepherd, J. M. (2005). A review of current investigations of urban-induced rainfall and recommendations for the future. Earth Interactions, 9(12), 1-27. 
653 Spalart, P. R. (1988). Direct Simulation of a Turbulent Boundary Layer up to Re_ $\theta=1410$. Journal

654 of Fluid Mechanics, 187(December 1986), 61-98.

655 Tseng, Y.-H., Meneveau, C., \& Parlange, M. B. (2006). Modeling Flow around Bluff Bodies and 656 Predicting Urban Dispersion Using Large Eddy Simulation. Environmental Science \& 657 Technology, 40(8), 2653-2662.

658 Venko, S., Vidal De Ventós, D., Arkar, C., \& Medved, S. (2014). An experimental study of natural and mixed convection over cooled vertical room wall. Energy and Buildings, 68(PARTA),

661

\section{Appendix A: sensitivity to street resolution}

We tested the sensitivity of the results to the resolution of each street (the distance between

664 blocks) for the advection regime (case 5) and the convection regime (case 7). Three cases were

665 simulated with a different number of grid points for each street: 1 (as in low resolution simulations

666 of table 2), 3 (as in high resolution simulations of table 1), and 5 (highest resolution). Note that the

667 total area of heat emission equals the area of the city and is the same for all cases. Figure 16 shows

668 maps of $u$ for the advection-dominated regime with three different street resolutions. It can be

669 noted that, while $u$ for lower street resolutions is slightly smaller at the upstream edge of the city

670 and behind the city (in the recirculation regime), overall the three cases show similar flow patterns.

671 Flow blockage (pressure drag) is thus slightly stronger at lower resolutions. Similarly, Figure 17

672 shows maps of $u$ for the convection-dominated regime with three different street resolutions. We

673 can see that circulations over the city in these cases are not very sensitive to the street resolutions.

674 In both cases, the large-scale flow patterns that we are examining in this paper are not affected. 
No. of grids $=1$

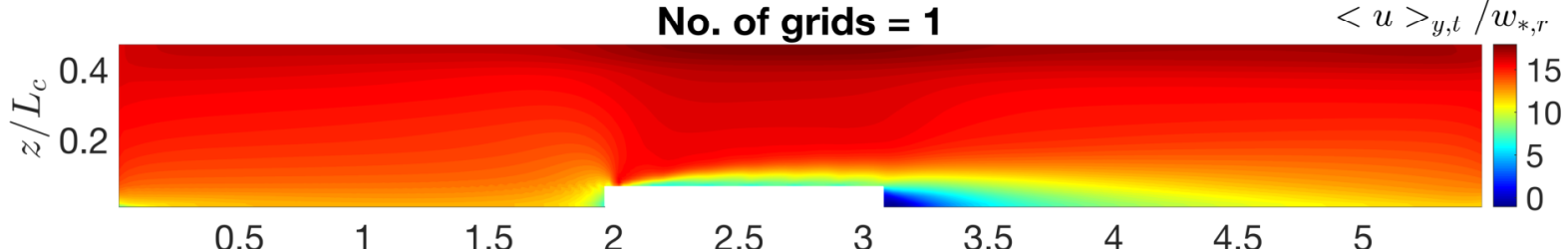

No. of grids $=3$

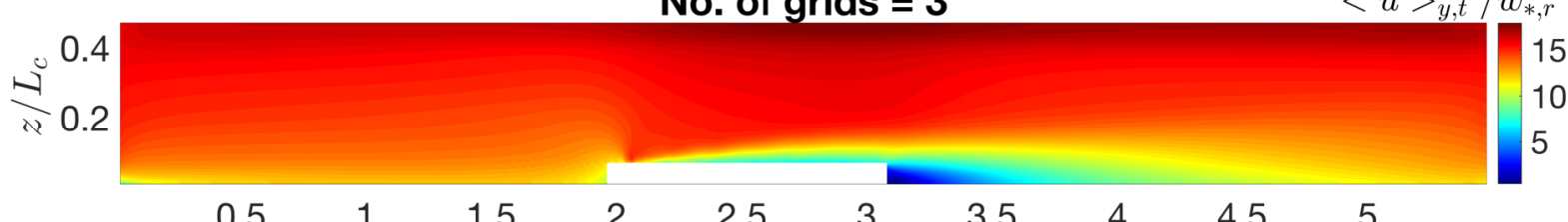

No. of grids $=5$

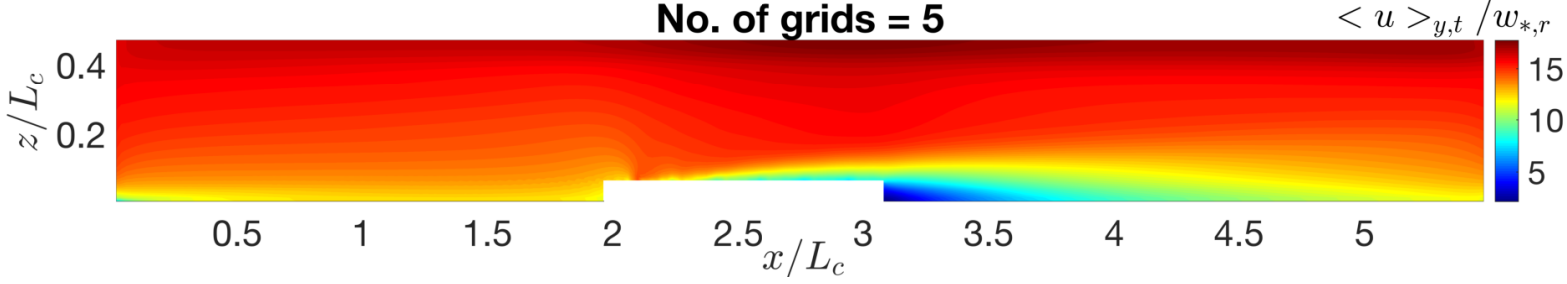

676 Figure 16. Pseudocolor plots of normalized (by average inflow) $u$ for advection dominated regime (case

677 5) with three different street resolutions. The white masked area contains both city blocks and (solid 


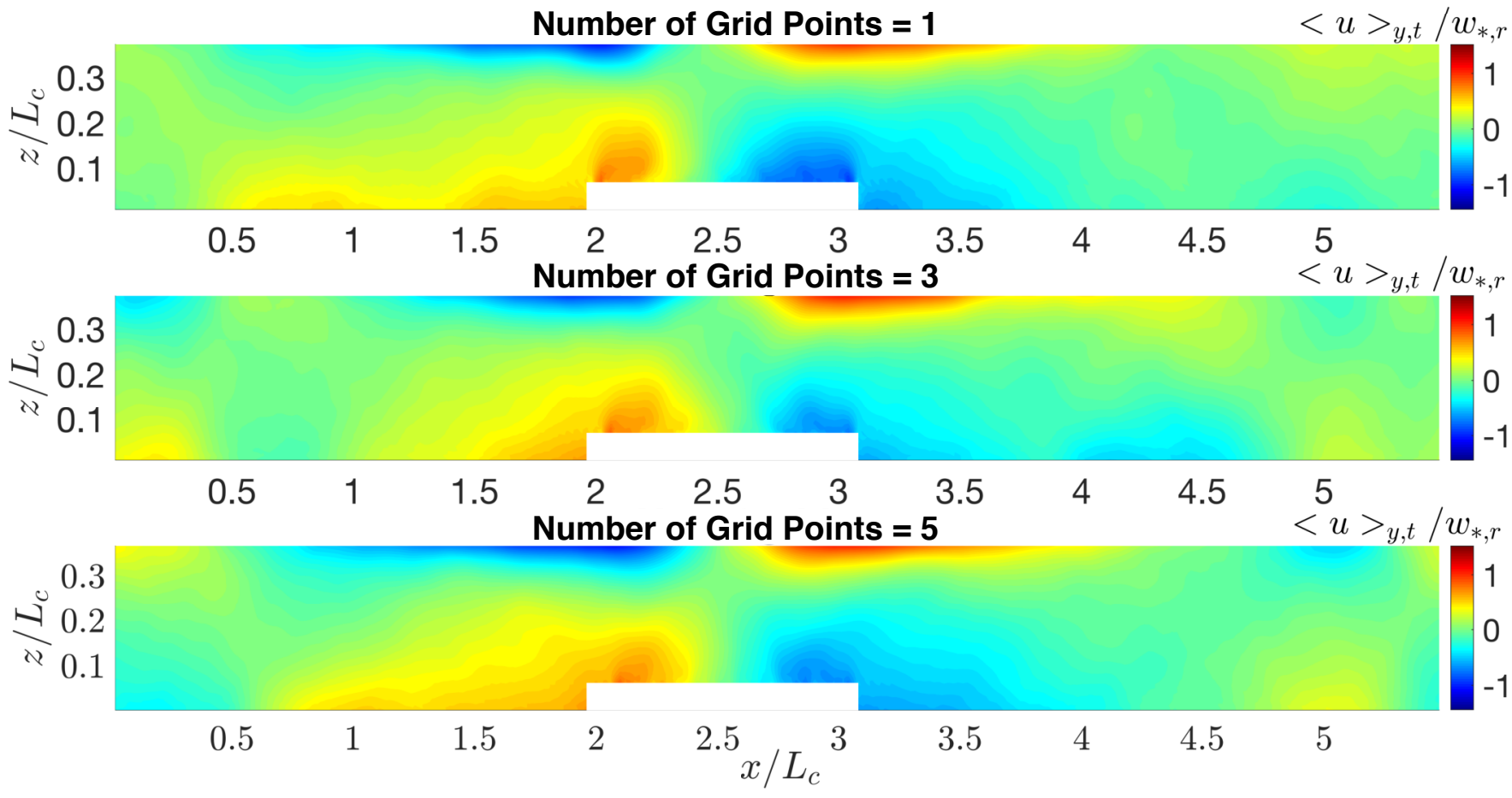

Figure 17. Pseudocolor plots of normalized (by $w *_{r}$ ) $w$ for convection dominated regime (case 7) with three different street resolutions.

\section{Appendix B: precursor runs and rescaling of the inflow}

684 The domain for the precursor runs has the same height (z-direction) and width ( $y$-direction) as the

685 main runs domain (Figure 2); however, the domain length for precursor runs is smaller than the

686 main domain $(3.5 \mathrm{~km}$, with the same resolution as the main domain in all directions). Similar to

687 the main domain, in inversion layer for the precursor domain covers the top $20 \%$ of the domain

688 height (and is maintained using the same approach as the main domain discussed in Section 3.1).

689 Therefore, the boundary layer height for the precursor runs is identical to the main domain and

690 kept constant at $80 \%$ of the domain height. The inflow variables (velocities and temperature) for

691 the main domain runs were extracted from the end point (an $y$-z slice) of the precursor runs domain. 
692 The inflow extraction is only started after the averaged TKE of the precursor domain reaches 693 steady state conditions.

694 For velocities, we can write the following relationships for the bulk velocity magnitudes

695 for the re-scaled precursor (with subscript $p$ ), and the main precursor that is actually simulated 696 (with subscript $m$ ) using MOST:

$$
\begin{aligned}
& \frac{M_{p}}{u_{*, p}}=\frac{1}{\kappa}\left[\ln \left(\frac{z}{z_{0, r}}\right)+\Psi_{M}\left(\frac{z}{L_{p}}\right)\right], \\
& \frac{M_{m}}{u_{*, m}}=\frac{1}{\kappa}\left[\ln \left(\frac{z}{z_{0, r}}\right)+\Psi_{M}\left(\frac{z}{L_{m}}\right)\right],
\end{aligned}
$$

699 where $z_{0, r}$ is the momentum roughness length of the rural area, $L$ the Obukhov length scale and

$700 \Psi_{M}$ the MOST stability function for momentum. If we approximate $\Psi_{M}\left(z / L_{p}\right) \approx \Psi_{M}\left(z / L_{m}\right)$,

701 which is plausible given that the heat flux influencing $L_{p}$ and $L_{m}$ is the same although the friction

702 velocities are different, then $M_{p}$ can be calculated by re-scaling the average of the generated main

703 inflow in the main precursor simulation to get the desired inflow as follows:

$$
M_{p}=\frac{u_{*, p}}{u_{*, m}} M_{m} .
$$

705 The same scaling is then used to generate the whole inflow planes for $u, v$, and $w$ as functions of $706 y, z$, and $t$. Using equation (9), we are able to use one generated inflow velocity for each of the 707 rural heat fluxes to produce a range of precursor inflows with different bulk averaged velocities.

708 For temperature, we can also invoke MOST to write the temperature profile for precursor 709 simulations and main runs as follows: 


$$
\begin{aligned}
& \theta_{s}^{\text {inflow }}-\theta_{m}^{\text {inflow }}=\frac{1}{\kappa} \frac{\left(\overline{\theta^{\prime} w^{\prime}}\right)_{r}}{u_{*, m}}\left[\ln \left(\frac{z}{z_{0, h}^{r}}\right)+\Psi_{\theta}\left(\frac{z}{L_{p}}\right)\right], \\
& \theta_{s}^{\text {inflow }}-\theta_{p}^{\text {inflow }}=\frac{1}{\kappa} \frac{\left(\overline{\theta^{\prime} w^{\prime}}\right)_{r}}{u_{*, p}}\left[\ln \left(\frac{z}{z_{0, h}^{r}}\right)+\Psi_{\theta}\left(\frac{z}{L_{p}}\right)\right],
\end{aligned}
$$

712 where $z_{0, h}^{r}=0.1 z_{0, m}^{r}$ is the heat roughness length of the rural area, and $\theta_{s}^{\text {inflow }}$ is the surface

713 temperature for the inflow that is assumed to be equal for both precursor and main simulations.

714 Assuming $\frac{\Psi_{\theta}\left(z / L_{p}\right)}{M_{p}} \approx \frac{\Psi_{\theta}\left(z / L_{m}\right)}{M_{m}}$ and subtracting equation (11) from (10), we obtain the

715 following re-scaling relation between the temperatures of the precursor and main simulations:

$$
\theta_{p}^{\text {inflow }}=\theta_{m}^{\text {inflow }}+\frac{1}{\kappa}\left(\overline{\theta^{\prime} w^{\prime}}\right)_{r} \ln \left(\frac{z}{z_{0 . h}^{r}}\right)\left(\frac{1}{M_{m}}-\frac{1}{M_{p}}\right)
$$

718 We reiterate that this rescaling need not be exact since (i) the inflow is allowed to evolve over a

719 distance $\approx 5$ times the boundary layer depth $(1800 \mathrm{~m} / 333 \mathrm{~m})$ inside the main domain to further

720 adjust to the upstream rural surface before it meets the city, and (ii) regardless of the rescaling

721 results, the $M$ used in the analyses is the one actually attained and computed just upstream of the 722 city in the main domain.

\section{Appendix C: sensitivity to the domain size}

Figure 18 shows the maps of the cross-stream velocity $v$ in the $z-y$ plane for three different values of $L_{y} / L_{c}=3.8,5$, and 5.6 (they correspond to $L_{y}=3330,4500$, and $5000 \mathrm{~m}$ ). All three simulations are conducted for the case without inflow (case 7 in Table 1). This case corresponds to the largest circulations around the city; therefore, we can use it to investigate the minimum 
domain size needed to prevent circulations from strongly interacting with each other across the periodic boundaries. One can observe that in the case of $L_{y} / L_{c}=3.8\left(L_{y}=3330 \mathrm{~m}\right)$, the circulations clearly interact with the left and right boundaries, and this leads to a right shift in the position of the circulations above the city (this could have as well been a deflection to the left). On the other hand, for the other two cases $\left(L_{y} / L_{c}=5\right.$ and 5.6), the horizontal extent of the circulations on either side of the city is roughly equal to 3 times the city-size; hence, the size of the domain in these cases is large enough to prevent the city circulations from directly interacting and does not affect the circulation scale. The figure also shows that indirect interaction through intermediate circulations are weak since these intermediate structures are less energetic. For all of the simulations in Table 1, we chose the intermediate domain size where $L_{y} / L_{c}=5\left(L_{y}=4500 \mathrm{~m}\right)$.
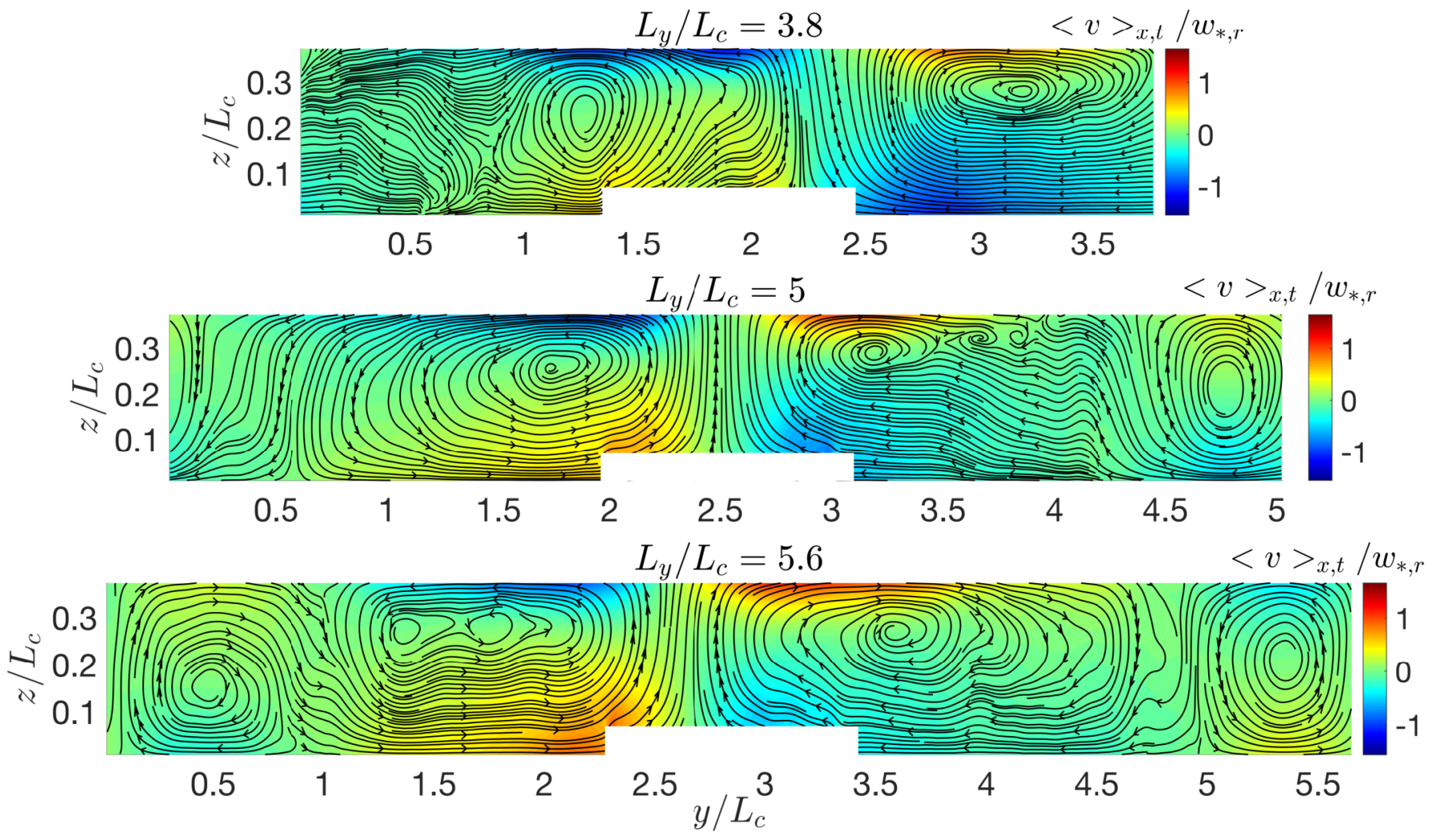

Figure 18. Pseudocolor maps of normalized (by $w_{*_{r}}$ ) $v$ in the $z-y$ plane for convection dominated regime (case 7) with three different $L_{y} / L_{c}: 3.8$ (top), 5 (middle), 5.6 (bottom). 


\section{Appendix D: Turbulent kinetic energy}

Figure 19 displays the horizontal profile of normalized (resolved) TKE for different regimes (transitional, plume and bubble). Similar to the mean velocities, the higher order statistics also follow the scaling similarity derived in this paper. For the transitional regime, cases 1 and 2 show similar TKE profiles that are distinct from those in cases 3 and 4 . Similar conclusion can be drawn for plume (cases 5 and 6 ) and bubble regimes (cases 7 and 8 ), not shown here.

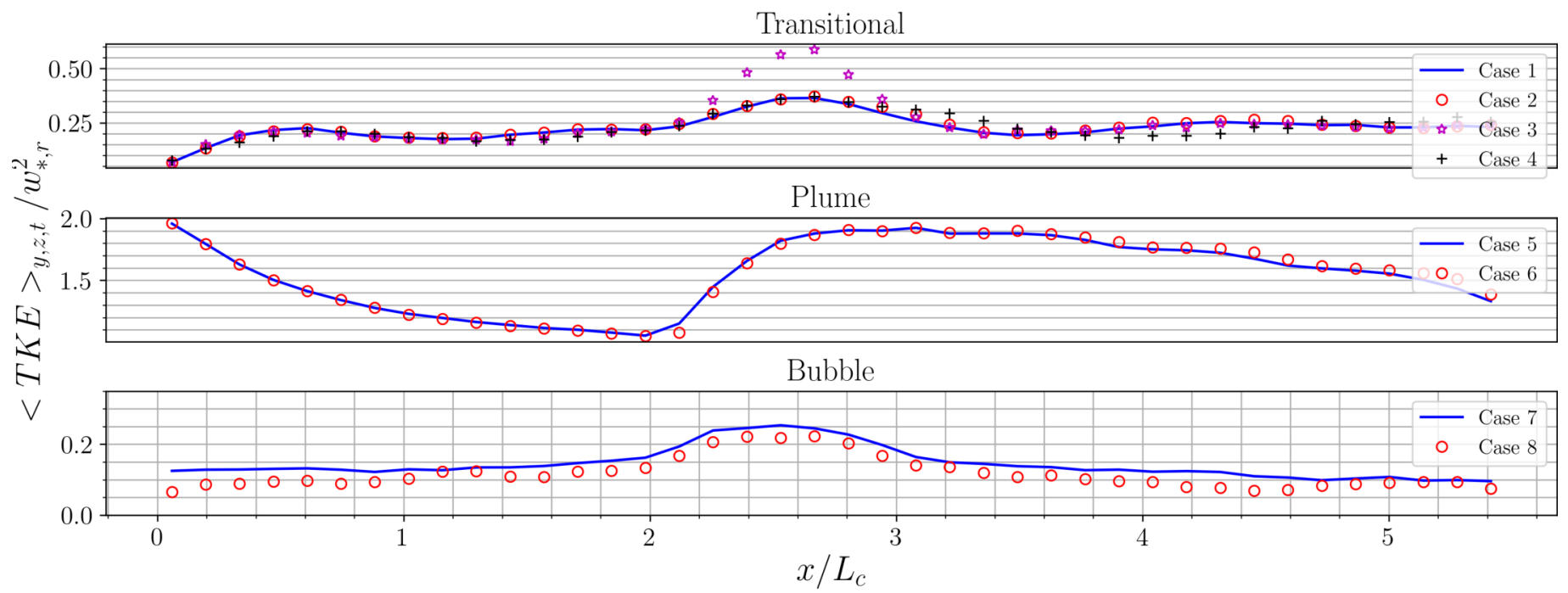

Figure 19. Horizontal profile of normalized (by $w^{2} *_{r}$ ) TKE for transitional (top panel), plume (middle panel) and bubble (bottom panel) regimes. 